\title{
Finite-volume energy spectrum, fractionalized strings, and low-energy effective field theory for the quantum dimer model on the square lattice
}

\author{
D. Banerjee, ${ }^{1,2}$ M. Bögli, ${ }^{1,3}$ C. P. Hofmann, ${ }^{4}$ F.-J. Jiang, ${ }^{5}$ P. Widmer, ${ }^{1}$ and U.-J. Wiese ${ }^{1}$ \\ ${ }^{1}$ Albert Einstein Center for Fundamental Physics, Institute for Theoretical Physics, \\ Bern University, Sidlerstrasse 5, CH-3012 Bern, Switzerland \\ ${ }^{2}$ NIC, DESY, Platenenallee 6, 15738 Zeuthen, Germany \\ ${ }^{3}$ Department of Physics, Chung-Yuan Christian University (CYCU), Chung-Li 32023, Taiwan \\ ${ }^{4}$ Facultad de Ciencias, Universidad de Colima, Colima C.P. 28045, Mexico \\ ${ }^{5}$ Department of Physics, National Taiwan Normal University, 88, Sec. 4, Ting-Chou Rd., Taipei 116, Taiwan
}

(Received 15 January 2016; published 8 September 2016)

\begin{abstract}
We present detailed analytic calculations of finite-volume energy spectra, mean-field theory, as well as a systematic low-energy effective field theory for the square lattice quantum dimer model. An emergent approximate spontaneously broken $\mathrm{SO}(2)$ symmetry gives rise to a pseudo-Goldstone boson. Remarkably, this soft phononlike excitation, which is massless at the Rokhsar-Kivelson (RK) point, exists far beyond this point. The Goldstone physics is captured by a systematic low-energy effective field theory. We determine its low-energy parameters by matching the analytic effective field theory with exact diagonalization results. This confirms that the model exists in the columnar (and not in a plaquette or mixed) phase all the way to the RK point.
\end{abstract}

DOI: 10.1103/PhysRevB.94.115120

\section{INTRODUCTION}

Despite the extensive work on high-temperature superconductivity during the past decades since their discovery [1], understanding the mechanism of electron or hole pairing still represents a major unsolved problem in condensed matter physics. One of the various proposed scenarios is related to the quantum dimer model that was introduced by Rokhsar and Kivelson in Ref. [2]. It represents a simple realization of the resonating valence bond (RVB) state, proposed by Anderson in his pioneering paper [3], and provides a possible route towards understanding high-temperature superconductivity. Quantum dimer models have attracted a lot of attention over the years, as they are also relevant beyond high-temperature superconductivity, e.g., in connection with deconfined quantum criticality or topological order. Unraveling the phase structure of both the classical and the quantum dimer model has been the subject of many publications [4-16].

These studies include dimer models on both bipartite and nonbipartite lattices, which are defined in spatial dimensions $d \geqslant 2$. Quite surprisingly, even in the case of the simple square lattice the question of which phases are realized as a function of the Rokhsar-Kivelson (RK) parameter $\lambda$ has been controversial. This may even be more surprising in view of the fact that Monte Carlo simulations of quantum dimer models on the square lattice are not affected by the sign problem. While some authors claimed that a plaquette phase arises from a columnar phase in a first-order phase transition around $\lambda \approx 0.6$ [17], other studies found evidence for a mixed phase for $\lambda \gtrsim 0$, exhibiting features of both the columnar and the plaquette phase [18].

In a recent study [19], using quantum Monte Carlo applied to dual height variables as well as exact diagonalization, we have challenged these various views. In particular, we pointed out that there is no evidence for a plaquette or mixed phase in the square lattice quantum dimer model — rather the columnar phase extends all the way to the RK point at $\lambda=1$. Moreover, we showed that two external static charges \pm 2 are confined by an electric flux string that fractionalizes into eight strands carrying fractionalized flux $\frac{1}{4}$. Inside these strands, which represent interfaces separating different columnar orders, we found plaquette phase. However, the plaquette phase only exists inside the strands and not in the bulk. Finally, as a consequence of an approximate emergent $\mathrm{SO}(2)$ symmetry, we found evidence for a soft pseudo-Goldstone boson that exists in the parameter regime $0 \lesssim \lambda<1$, i.e., even far beyond the RK point.

In the present article we complement our previous Monte Carlo and exact diagonalzation results with detailed analytic calculations of finite-volume energy spectra, mean-field theory, as well as a systematic low-energy effective field theory for the pseudo-Goldstone boson. Overall, we consolidate our previous findings that contradict the earlier views on the phase structure of the square lattice quantum dimer model. We would like to point out that many of the analytical results of the present manuscript never appeared in Ref. [19], hence are new. In particular, one new Monte Carlo result with intriguing physics is shown in present manuscript as well.

The paper is organized as follows. In section II we define the quantum dimer model and discuss its symmetries on the square lattice. We then introduce height variables on the dual lattice, which have already been introduced in Ref. [19]. Here we give a more detailed description, showing how to construct these height variables, to make the current manuscript selfcontained. Notice on the one hand, these allow us to define order parameters that distinguish the various candidate phases. On the other hand, the dual height variables are the basic degrees of freedom on which the Monte Carlo simulations shown in Ref. [19] operate. We also emphasize the difference of our height variables and those conventionally used in the literature, since it forms the very basis of designing the order parameters used to study the system. This would, for example, also be useful for studying similar phases in other lattice Hamiltonians, and is of more general interest than the dimer model alone. Finally, in Sec. II, a systematic mean-field analysis of the quantum dimer model is conducted 

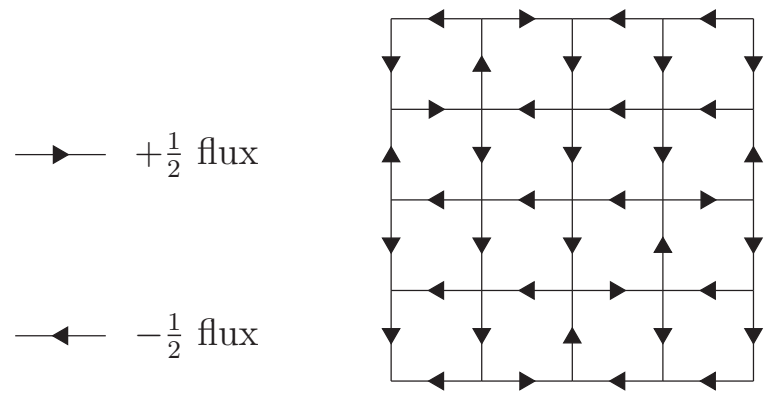

FIG. 1. Definition of flux states (left) and typical flux configuration on the square lattice U(1) quantum link model (right).

as well. In Sec. III, we investigate the finite-volume energy spectrum as a diagnostic of the phase structure. Section IV is dedicated to the low-energy effective field theory for the soft pseudo-Goldstone mode and the corresponding rotor spectrum. Some topics discussed in Secs. III and IV are reproduced from Ref. [19] for the benefit of the readers. In Sec. V, we present exact diagonalization results and use them to estimate some low-energy parameters of the effective field theory. In Sec. VI, we present new Monte Carlo data for the confining strings in the columnar phase. Finally, in Sec. VII, we present our conclusions. An appendix summarizes the symmetry properties of the relevant order parameters.

\section{MODEL AND OBSERVABLES}

In this section, we consider the quantum dimer model and discuss its symmetries on the square lattice. We then define height variables on the dual lattice, which are the basic degrees of freedom in our Monte Carlo simulations. They also serve to construct order parameters that signal which phase is realized. Finally, we perform a systematic mean-field analysis with the intention to gain qualitative insight into this question.

\section{A. Model}

The Hamiltonian of the quantum dimer model coincides with the Hamiltonian of the $(2+1)-d U(1)$ quantum link model [20-22]. However, the corresponding Gauss law is realized differently. The Hamiltonian of both the U(1) quantum link model and the quantum dimer model takes the form

$$
H=-J \sum_{\square}\left[U_{\square}+U_{\square}^{\dagger}-\lambda\left(U_{\square}+U_{\square}^{\dagger}\right)^{2}\right] .
$$

In the above Hamiltonian, the quantity $U_{\square}=U_{w x} U_{x y} U_{z y}^{\dagger} U_{w z}^{\dagger}$ represents a plaquette operator expressed in terms of quantum links $U_{x y}$ that connect the nearest-neighbor sites $x$ and $y$ on the square lattice. A $\mathrm{U}(1)$ quantum link $U_{x y}=S_{x y}^{+}$is a raising operator of the electric flux $E_{x y}=S_{x y}^{3}$, which is built from a quantum spin $\frac{1}{2}$ associated with the link $x y$. In the $\mathrm{U}(1)$ quantum link model, each link has two possible states characterized by electric flux $\pm \frac{1}{2}$, represented pictorially by arrows as shown in Fig. 1. A typical flux configuration of the $\mathrm{U}(1)$ quantum link model is depicted in the same figure.

Applying the Hamiltonian of Eq. (2.1) to a plaquette flux state leads to the results shown in Fig. 2. In summary, the first contribution to the Hamiltonian (2.1), proportional to the
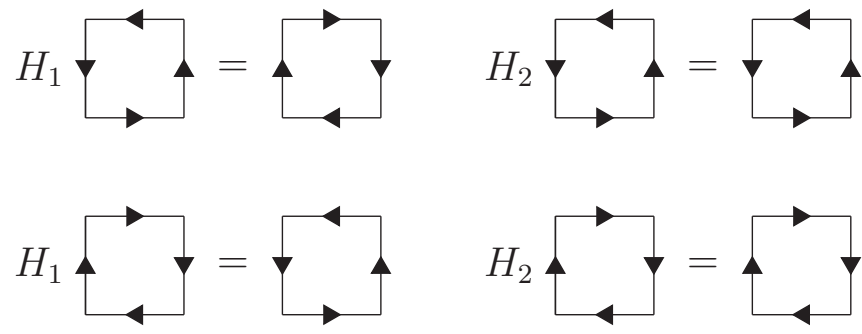

FIG. 2. The results of applying the Hamiltonian of Eq. (2.1) to some plaquette flux states. Here, $H_{1}$ and $H_{2}$ represent the terms in Eq. (2.1) proportional to $J$ and $J \lambda$, respectively. When the Hamiltonian acts on other plaquette configurations (which are not shown explicitly) the result vanishes.

parameter $J$, flips a loop of flux that winds around a plaquette. Flux states that do not correspond to closed flux loops are referred to as nonflippable plaquettes, which are annihilated by the Hamiltonian. On the other hand, the second contribution to the Hamiltonian (2.1), proportional to the RK parameter $\lambda$, counts the plaquettes that are flippable. Notice that the configurations of the square lattice quantum dimer model are characterized in terms of variables $D_{x y} \in\{0,1\}$, which signal whether a dimer is present or absent on the link that connects two neighboring sites $x$ and $y$. In addition, the electric flux variables $E_{x y}$ can be expressed through the dimer variables $D_{x y}$ as

$$
E_{x y}=(-1)^{x_{1}+x_{2}}\left(D_{x y}-\frac{1}{2}\right) .
$$

This mapping between a dimer and a flux configuration of the quantum dimer model is illustrated in Fig. 3.

Notice that, in the U(1) quantum link model, the physical state $|\psi\rangle$ satisfies

$$
G_{x}|\psi\rangle=0
$$

where the quantity

$$
G_{x}=\sum_{i}\left(E_{x, x+\hat{i}}-E_{x-\hat{i}, x}\right)
$$

commutes with the Hamiltonian and describes an infinitesimal $\mathrm{U}(1)$ gauge transformation. Here, $\hat{i}$ is the unit vector in the $i$ direction. Equation (2.3) represents the Gauss law for the U(1) quantum link model. In the quantum dimer model, using the connection between the electric flux and the dimer variables,
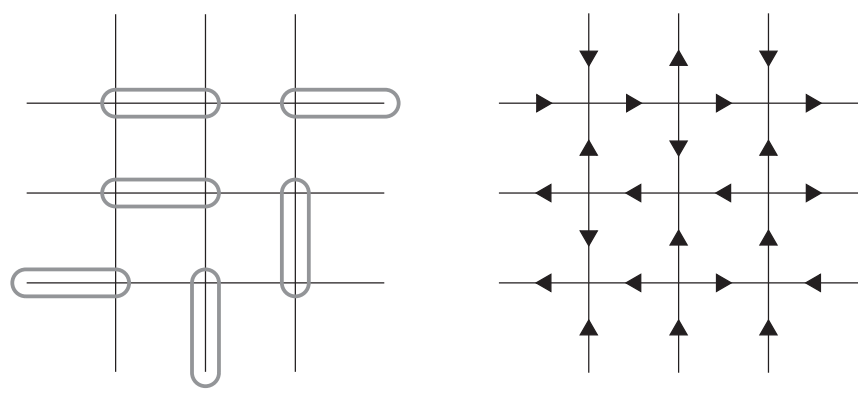

FIG. 3. Mapping between dimer and flux configurations. 
one has

$$
G_{x}=(-1)^{x_{1}+x_{2}} \sum_{i}\left(D_{x, x+\hat{i}}+D_{x-\hat{i}, x}\right)=(-1)^{x_{1}+x_{2}} .
$$

In other words, the dimer covering constraint implies that the quantum dimer model is characterized by background electric charges \pm 1 that are arranged in a staggered pattern. Accordingly, physical states in the quantum dimer model satisfy

$$
G_{x}|\Psi\rangle=(-1)^{x_{1}+x_{2}}|\Psi\rangle
$$

\section{B. Symmetries}

The quantum dimer model on the square lattice exhibits various symmetries. We first have a continuous U(1) gauge symmetry and a global $\mathrm{U}(1)^{2}$ center symmetry. The latter is associated with "large" gauge transformations [23]. The model also has various discrete global symmetries. These include translations by one lattice vector followed by charge conjugation $\left(C T_{x}\right.$ and $\left.C T_{y}\right)$, which are equivalent to ordinary translations of the dimers $D_{x y}$. Note that charge conjugation changes the sign of all electric flux variables. It is important to point out that, in contrast to the quantum link model, the transformations $T_{x}, T_{y}$, and $C$ individually are not symmetries of the quantum dimer model because they are explicitly violated by the Gauss law. Furthermore, we have $90^{\circ}$ rotations around a plaquette corner $(O), 90^{\circ}$ rotations around a plaquette center followed by charge conjugation $\left(C O^{\prime}\right)$, and finally, reflections on the $x$ and $y$ axes $\left(R_{x}\right.$ and $\left.R_{y}\right)$. Below we will construct order parameters that will help us to determine which phases are realized in the square lattice quantum dimer model. It is then crucial to know how these different order parameters transform under the various symmetries (see Sec. II D and Appendix).

\section{Dual height variables}

In this section, we introduce height variables that reside on the dual lattice. This height representation of the quantum dimer model is essential in our approach. It allows us, on the one hand, to design cluster and Metropolis algorithms that operate in the space of these height variables and, on the other hand, to construct order parameters to unambiguously distinguish the various phases.

As illustrated in Fig. 4, in the case of the square lattice quantum dimer model, we define four dual sublattices $A, B, C$, and $D$, which consist of the points

$$
\tilde{x}=\left(x_{1}+\frac{1}{2}, x_{2}+\frac{1}{2}\right) .
$$

Each of the dual sublattices $X$ carries dual height variables $h^{X}$ that take the values

$$
h_{\widetilde{x}}^{A, D}=0,1, \quad h_{\tilde{x}}^{B, C}= \pm \frac{1}{2} .
$$

When defining the height variables on the dual lattice, we will encounter an additional complication compared to the U(1) quantum link model, which is due to the fact that the Gauss law is realized differently in these two models. This further complication requires the introduction of so-called Dirac strings, in order to consistently relate the height variables $h_{\widetilde{x}}^{X}$ with the electric fluxes $E_{x, y}$. These Dirac strings are located

\begin{tabular}{|c|c|c|c|c|c|}
\hline $\mathrm{A}$ & $\mathrm{B}$ & $\mathrm{A}$ & $\mathrm{B}$ & $\mathrm{A}$ & $\mathrm{B}$ \\
\hline $\mathrm{C}$ & $\mathrm{D}$ & $\mathrm{C}$ & $\mathrm{D}$ & $\mathrm{C}$ & $\mathrm{D}$ \\
\hline $\mathrm{A}$ & $\mathrm{B}$ & $\mathrm{A}$ & $\mathrm{B}$ & $\mathrm{A}$ & $\mathrm{B}$ \\
\hline $\mathrm{C}$ & $\mathrm{D}$ & $\mathrm{C}$ & $\mathrm{D}$ & $\mathrm{C}$ & $\mathrm{D}$ \\
\hline $\mathrm{A}$ & $\mathrm{B}$ & $\mathrm{A}$ & $\mathrm{B}$ & $\mathrm{A}$ & $\mathrm{B}$ \\
\hline $\mathrm{C}$ & $\mathrm{D}$ & $\mathrm{C}$ & $\mathrm{D}$ & $\mathrm{C}$ & $\mathrm{D}$ \\
\hline
\end{tabular}

FIG. 4. The four dual sublattices $A, B, C$, and $D$ used in the construction of the height variables $h^{A, B, C, D}$.

in a staggered fashion on the vertical links and are denoted by empty squares on the links (see Fig. 5).

The quantities $h^{A, B, C, D}$, residing at the sites of a dual sublattice, are related to the electric flux variables on the links by

$$
\begin{aligned}
E_{x, x+\hat{1}} & =\left[h_{\widetilde{x}}^{X}-h_{\widetilde{x}-\hat{2}}^{X^{\prime}}\right] \bmod 2= \pm \frac{1}{2}, \\
E_{x, x+\hat{2}} & =(-1)^{x_{1}+x_{2}}\left[h_{\widetilde{x}}^{X}-h_{\widetilde{x}-\hat{1}}^{X^{\prime}}\right] \bmod 2= \pm \frac{1}{2}, \\
X, X^{\prime} & \in\{A, B, C, D\} .
\end{aligned}
$$

Note that whenever $(-1)^{x_{1}+x_{2}}=-1$, it indicates the presence of a Dirac-string on the relevant vertical link. The corresponding height representation and the flux representation for a columnar dimer configuration is illustrated in Fig. 5. Beside the height and flux variables, we have also marked positive and negative background charges (filled and empty circles) as well as the Dirac strings (empty squares).

It should be noted that this construction of height variables is new and fundamentally different from other height variables definitions that have been introduced in the literature, in particular, the one described in the review paper of Moessner and Raman [24]. In addition, there is no obvious connection between our height variables and that built by Zheng and Sachdev as well [25]. For instance, in our construction the height variables take only four values: 0,1 , and $\pm 1 / 2$. On the
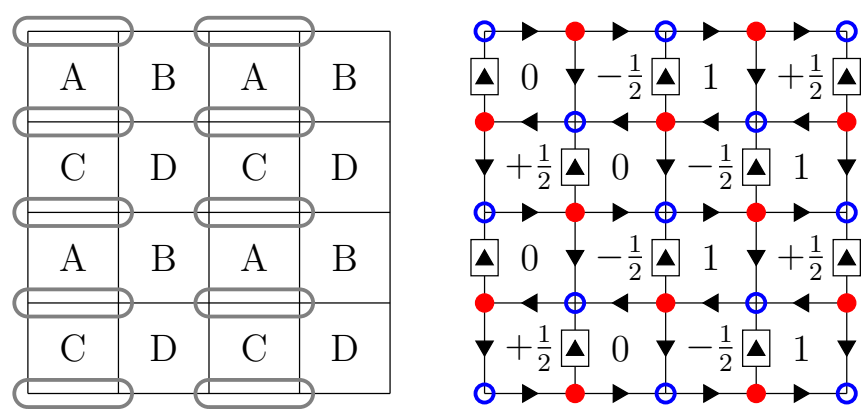

FIG. 5. The mapping between the dimer configuration and the corresponding flux and height representation for a columnar quantum dimer configuration. 
(a)

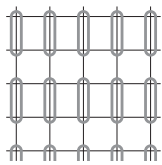

(b)

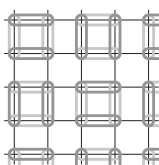

(c)

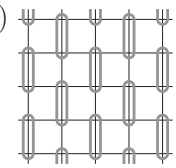

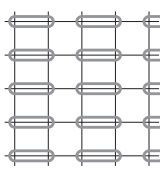
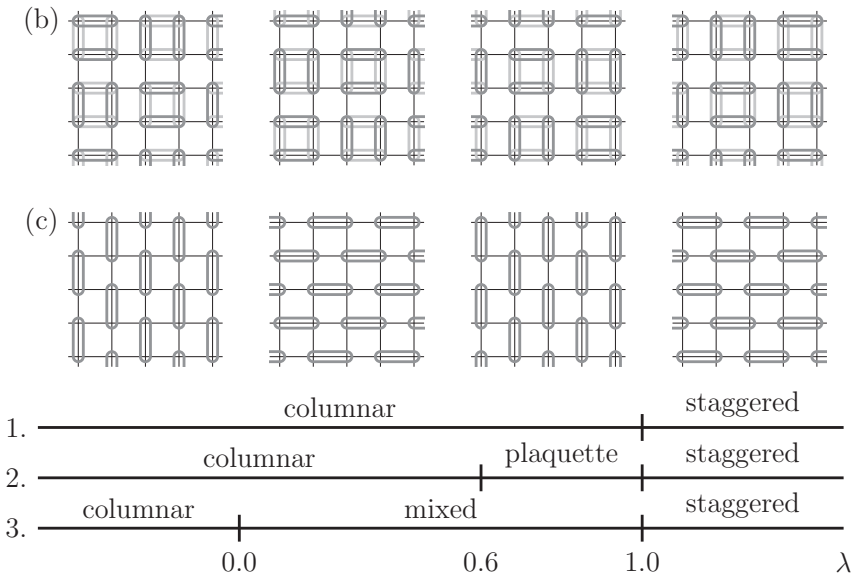

FIG. 6. Established and conjectured phases for the square lattice quantum dimer model: (a) Columnar, (b) plaquette, (c) staggered order on the dual sublattices $A, B, C$, and $D$. (d) Phase diagram for the square lattice quantum dimer model as a function of the RK parameter $\lambda$ : three different scenarios. This figure is reproduced from Ref. [19] for the benefit of the readers.

other hand, with the conventional methods they are always labeled with integers and can take many more values. Our height variables take much fewer values than the conventional ones, because they provide an exact representation of the dimer model Hilbert space.

\section{Order parameters and candidate phases}

In this section, we review four order parameters in terms of the height variables that we introduced in [19]. Each of the four candidate phases-staggered, columnar, plaquette or mixed - can then be unambiguously identified by the specific values these order parameters take in the different phases.

Let us first discuss the various phases that have been established or conjectured for the square lattice quantum dimer model. Intuitively, in the limit $\lambda \rightarrow-\infty$, the system maximizes the number of flippable plaquettes. On the square lattice, such a state can be obtained by arranging the dimers in a columnar pattern as depicted in Fig. 6(a). Note that we are dealing with fourfold degeneracy: the four columnar configurations are related by translations or rotations.

On the other hand, in the opposite limit $\lambda \rightarrow+\infty$, the system minimizes the number of flippable plaquettes. On the square lattice, such a state can be obtained by arranging the dimers in a staggered pattern, shown in Fig. 6(c). The four degenerate staggered phases are related by discrete transformations.

Another candidate phase is the so-called plaquette arrangement of dimers, which is also fourfold degenerate, and is illustrated in Fig. 6(b). In this phase, pairs of parallel dimers oriented in both possible directions resonate on the plaquettes belonging to one of the four sublattices $A, B, C$, and $D$.
Finally, on the square lattice, another conjectured phase is the so-called mixed phase which is eightfold degenerate and corresponds to a superposition of quantum dimer states, sharing features of both the columnar and the plaquette phase.

Apart from the established columnar and staggered phases in the limits $\lambda \rightarrow-\infty$ and $\lambda \rightarrow+\infty$, respectively, the question of which phases are realized between these two points of reference-and what type of associated phase transitions might exist - has remained controversial. In Ref. [19], we have challenged the various conflicting scenarios that have been proposed in earlier studies $[17,18]$ and are depicted in Fig. 6(d). An important point of reference is the $\operatorname{RK}$ point $(\lambda=1)$ where the model is exactly solvable. Away from the RK point, the situation becomes less clear. Using Green's function Monte Carlo simulations, the author of Ref. [17] concludes that on the square lattice there is a phase transition between the columnar and plaquette phase around $\lambda \approx 0.6$ [scenario 2 in Fig. 6(d)]. However, this view is not shared by Ref. [18], which favors a mixed phase for $\lambda \gtrsim 0$ according to their Green's function Monte Carlo analysis [scenario 3 in Fig. 6(d)]. Based on our new order parameters and a novel Monte Carlo technique we concluded that the system exists in a columnar phase all the way up to the RK point [scenario 1 in Fig. 6(d)]. In particular, we found no evidence for plaquette or mixed phases.

For completeness, we now review the four order parameters whose construction is based on the dual height representation. Remember that we have two sets of height variables, the first one associated with the even sublattices $A$ and $D$, the second one related to the odd sublattices $B$ and $C$ (see Fig. 4).

We first define four auxiliary order parameters $M_{A}, M_{B}$, $M_{C}$, and $M_{D}$ as

$$
M_{X}=\sum_{\widetilde{x} \in X} s_{\widetilde{x}}^{X} h_{\widetilde{x}}^{X},
$$

with

$$
\begin{array}{llll}
s_{\widetilde{x}}^{A}=s_{\widetilde{x}}^{C}=(-1)^{\left(\widetilde{x}_{1}+\frac{1}{2}\right) / 2}, & \text { if } & \tilde{x}_{1}+\frac{1}{2} & \text { even, } \\
s_{\widetilde{x}}^{B}=s_{\widetilde{x}}^{D}=(-1)^{\left(\widetilde{x}_{1}-\frac{1}{2}\right) / 2}, & \text { if } & \widetilde{x}_{1}+\frac{1}{2} & \text { odd. }
\end{array}
$$

Remember that the height variables on the various sublattices take the values

$$
h_{\widetilde{x}}^{A, D}=0,1, \quad h_{\widetilde{x}}^{B, C}= \pm \frac{1}{2} .
$$

We then form the linear combinations:

$$
\begin{aligned}
& M_{11}=M_{A}-M_{B}-M_{C}+M_{D}=M_{1} \cos \varphi_{1}, \\
& M_{22}=M_{A}+M_{B}-M_{C}-M_{D}=M_{1} \sin \varphi_{1}, \\
& M_{12}=M_{A}-M_{B}-M_{C}-M_{D}=M_{2} \cos \varphi_{2}, \\
& M_{21}=-M_{A}+M_{B}-M_{C}-M_{D}=M_{2} \sin \varphi_{2},
\end{aligned}
$$

which define the order parameters $M_{11}, M_{12}, M_{21}$, and $M_{22}$ that are more appropriate to distinguish the phases. The two angles $\varphi_{1}$ and $\varphi_{2}$ define the angle

$$
\varphi=\frac{1}{2}\left(\varphi_{1}+\varphi_{2}+\frac{\pi}{4}\right) .
$$

In the columnar phase this angle amounts to $\varphi=0 \bmod \frac{\pi}{4}$, while in the plaquette phase it takes the value $\varphi=\frac{\pi}{8} \bmod \frac{\pi}{4}$. Note that the order parameter values $\pm\left(M_{A}, M_{B}, M_{C}, M_{D}\right)$, 


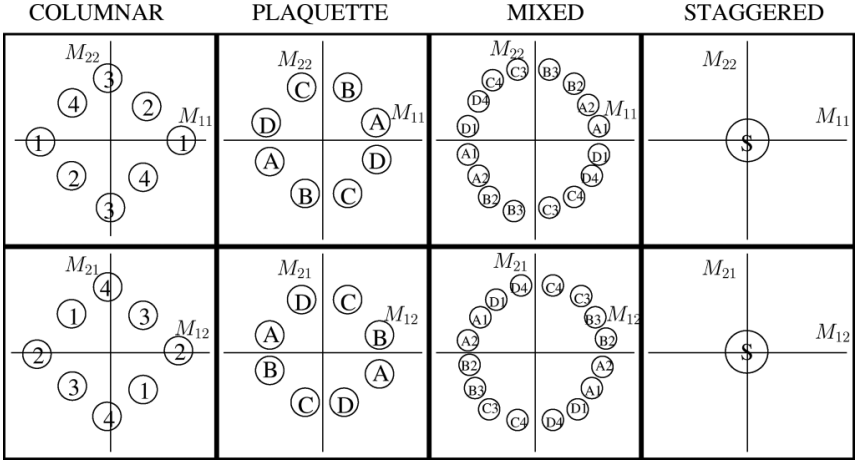

FIG. 7. The four candidate phases-columnar, plaquette, mixed, staggered-can unambiguously be distinguished by their characteristic order parameter distributions. This figure is reproduced from Ref. [19] for the benefit of the readers.

and therefore $\varphi$ and $\varphi+\pi$, represent the same physical dimer configuration, because a dimer configuration is invariant under a shift of the height variables,

$$
h_{\widetilde{x}}^{X}(t)^{\prime}=\left[h_{\widetilde{x}}^{X}(t)+1\right] \bmod 2 .
$$

As illustrated in Fig. 7, each of the four phases-columnar, plaquette, mixed, staggered-is characterized by its specific order parameter pattern. While there are four columnar phases $(1,2,3,4)$ and four plaquette phases $(\mathrm{A}, \mathrm{B}, \mathrm{C}$, and $\mathrm{D})$, there are eight realizations of the mixed phase (A1, A2, B2, B3, C3, C4, D4, D1). The mixed phases share features of both the columnar and the plaquette phases. For instance, in a hypothetical phase transition between a columnar and a mixed phase, a peak in the order parameter distribution of the columnar phase would split into two individual peaks: the columnar peak 1 would split into the peaks D1 and A1 referring to the mixed phase, etc. On the other hand, in a hypothetical phase transition between a mixed and a plaquette phase, two peaks in the order parameter distribution of the mixed phase would merge pairwise into one peak referring to the plaquette phase: the mixed peaks A1 and A2 would merge into the plaquette peak A, etc. As we will elaborate in more detail below, in our numerical simulations no such splitting or merging of peaks is detected.

For completeness, in an appendix, we show how the four order parameters transform under the symmetries $C T_{x}, C T_{y}, O, C O^{\prime}, R_{x}$, and $R_{y}$ of the square lattice quantum dimer model and how the different symmetries $C T_{x}, C T_{y}, O, C O^{\prime}, R_{x}$, and $R_{y}$ act on the columnar, plaquette, and mixed phases, respectively.

It should be pointed out that the specific phases that are actually realized, depend on both the lattice geometry and the spatial dimension. While we restrict ourselves to the (two-dimensional) square lattice, more complicated phases are indeed possible on other lattices. On nonbipartite lattices, in two or higher spatial dimensions, a $Z_{2}$ resonance valence bond liquid phase is formed [26-28]. This is a phase with $Z_{2}$ topological order, characterized by fourfold degenerate gapped ground states in the case of two-dimensional lattices with periodic boundary conditions. In particular, it has nontrivial excitations and represents a liquid phase because all dimer correlations decay exponentially. Another example is the U(1) resonating valence bond liquid phase that is possible on bipar- tite lattices and in spatial dimensions three or higher [29-31]. Even more complex phases include, e.g., the $\sqrt{12} \times \sqrt{12}$ phase, which appears to be realized on the triangular lattice $[28,32]$.

\section{E. Mean-field theory}

Using mean-field theory, in this section, we address the question which phases in the square lattice quantum dimer model may be realized in the vicinity of the RK point. Following the Ginsburg-Landau-Wilson paradigm, we formulate an effective action for the system in terms of the order parameters $M_{11}, M_{12}, M_{21}$, and $M_{22}$, defined in Eq. (2.13). The most general expression up to quartic order that respects all the symmetries of the underlying quantum dimer model, is given by

$$
\begin{aligned}
& V=\mu_{1} O_{1}+\mu_{2} O_{2}+v_{0} O_{1} O_{2}+\sum_{i=1}^{5} v_{i}\left|O_{i}\right|^{2}, \\
& O_{1}=M_{11}^{2}+M_{22}^{2}+M_{12}^{2}+M_{21}^{2}, \\
& O_{2}=M_{11} M_{12}-M_{11} M_{21}+M_{22} M_{12}+M_{22} M_{21}, \\
& O_{3}=M_{11}^{2}+M_{22}^{2}-M_{12}^{2}-M_{21}^{2}, \\
& O_{4}=M_{11} M_{12}+M_{11} M_{21}-M_{22} M_{12}+M_{22} M_{21}, \\
& O_{5}=M_{11} M_{22}+i M_{12} M_{21} .
\end{aligned}
$$

We have two quadratic and six quartic operators, i.e., a total of eight parameters $\mu_{1}, \mu_{2}, v_{0}, \ldots, v_{5}$. Each of the terms in the effective potential is invariant under the discrete symmetries, i.e., under $O, C O^{\prime}, R_{x}, R_{y}, C T_{x}$, and $C T_{y}$.

We perform a systematic analysis of the minima of the potential $V$ in the infinitesimal neighborhood of the staggered phase which begins at the RK point, and is characterized by $M_{11}=M_{12}=M_{21}=M_{22}=0$. Since the staggered phase corresponds to a stable minimum, we first diagonalize the mass squared matrix M:

$$
\begin{aligned}
& \mu_{1} O_{1}+\mu_{2} O_{2} \\
& \quad=\left(M_{11}, M_{12}, M_{21}, M_{22}\right) \mathbf{M}\left(M_{11}, M_{12}, M_{21}, M_{22}\right)^{T}
\end{aligned}
$$

near this point. All eigenvalues turn out to be positive if the two conditions

$$
\mu_{1}+\frac{\mu_{2}}{\sqrt{2}}>0, \quad \mu_{1}-\frac{\mu_{2}}{\sqrt{2}}>0,
$$

are satisfied. Assuming $\mu_{1}, \mu_{2}>0$, we obtain two zero eigenvalues if $\mu_{2}=\sqrt{2} \mu_{1}$. The corresponding eigenvectors $v_{1}$ and $v_{2}$ define the $x y$ plane of vectors $v$ parametrized by

$$
\left(\begin{array}{c}
\frac{x}{\sqrt{2}} \\
\frac{1}{2}(y-x) \\
\frac{1}{2}(y+x) \\
-\frac{y}{\sqrt{2}}
\end{array}\right) .
$$

This plane corresponds to the flat directions in which the staggered phase is about to become unstable. Let us therefore 
evaluate the quartic potential along these flat directions. The calculation shows that the potential can be reduced to the simple form

$$
\begin{aligned}
V(x, y)= & \left(\mu_{1}-\frac{\mu_{2}}{\sqrt{2}}\right)\left(x^{2}+y^{2}\right) \\
& +\left(-\frac{v_{0}}{\sqrt{2}}+v_{1}+\frac{v_{2}}{2}+\frac{v_{5}}{16}\right)\left(x^{2}+y^{2}\right)^{2} \\
= & \mu\left(x^{2}+y^{2}\right)+v\left(x^{2}+y^{2}\right)^{2} .
\end{aligned}
$$

The minima of the potential $V$ form a circle of radius $r=$ $\sqrt{x^{2}+y^{2}}$ with $r^{2}=-\mu / 2 v$. Parametrizing the vacuum circle by an angle $\phi$ as $x=r \cos \phi, y=r \sin \phi$, we get

$$
\left(\begin{array}{c}
\frac{r}{\sqrt{2}} \cos \phi \\
\frac{r}{2}(\sin \phi-\cos \phi) \\
\frac{r}{2}(\sin \phi+\cos \phi) \\
-\frac{r}{\sqrt{2}} \sin \phi
\end{array}\right) \text {. }
$$

We now derive general conditions for the minima of the full potential $V$ displayed in Eq. (2.16). After a lengthy, but otherwise trivial calculation, for the columnar phase, characterized by $M_{22}=0$ and $M_{21}=-M_{12}$, the potential at a columnar minimum amounts to

$$
V\left(M_{11}, M_{12},-M_{12}, 0\right)=\frac{\mu_{1}}{2}\left(M_{11}^{2}+2 M_{12}^{2}\right)+\mu_{2} M_{11} M_{12} .
$$

An analogous calculation for the plaquette phase, characterized by $M_{12}=M_{11}$ and $M_{21}=-M_{22}$, shows that the potential at a plaquette minimum corresponds to

$$
\begin{aligned}
& V\left(M_{11}, M_{11},-M_{22}, M_{22}\right) \\
& \quad=\mu_{1}\left(M_{11}^{2}+M_{22}^{2}\right)+\frac{\mu_{2}}{2}\left(M_{11}^{2}-M_{22}^{2}+2 M_{11} M_{22}\right) .
\end{aligned}
$$

However, it turns out that these two points, along with the six additional points that correspond to the other columnar and plaquette phases, all have the same energy on the circle of minima, Eq. (2.21). Hence, in order to decide which phasecolumnar or plaquette-is in fact favored, we have to perturb around these minima.

A stability analysis shows that there are indeed unstable directions, associated with negative eigenvalues of the mass squared matrix of the second derivatives. In fact, both the columnar and the plaquette phase can be associated with negative eigenvalues and the energy of both phases can be lowered by proceeding into the unstable directions. However, the relative energies of the phases reached in this way are very sensitive to the parameters $v_{i}$ that are unknown. The mean-field analysis hence does not lead to a conclusive answer of which phase - columnar or plaquette - is preferred near the RK point. Symmetries alone do not favor one of these two candidate phases over the other. We are thus dealing with a truly dynamical question which has to be explored with more elaborate methods such as Monte Carlo simulations. One may wonder whether a perturbation calculation in $\delta$ at the RK point will lead to a definite result of which phase(s) is (are) realized close to the RK point. We believe the mean-field analysis carried out here is more suitable than a perturbation expansion to address the stability of phases. Hence such a study based on a perturbation expansion at the RK point is left for future investigation.

\section{LOW-ENERGY SPECTRUM IN FINITE VOLUME}

In this section, we consider the lowest states in the finite-volume energy spectrum associated with the columnar, plaquette, and mixed phases, respectively. This will be useful for identifying the phase structure based on numerical results obtained by exact diagonalization studies.

\section{A. Low-energy spectrum in the columnar phase}

Let us first consider the finite-volume energy spectrum in the columnar phase. The four columnar phases give rise to four almost degenerate eigenstates, which can be chosen as simultaneous eigenstates of the $90^{\circ}$ rotation $O$ with eigenvalues $+1,-i,+i,-1$ as

$$
\begin{aligned}
& |+1\rangle=\frac{1}{2}(|1\rangle+|2\rangle+|3\rangle+|4\rangle), \\
& |-i\rangle=\frac{1}{2}(|1\rangle+i|2\rangle-|3\rangle-i|4\rangle), \\
& |+i\rangle=\frac{1}{2}(|1\rangle-i|2\rangle-|3\rangle+i|4\rangle), \\
& |-1\rangle=\frac{1}{2}(|1\rangle-|2\rangle+|3\rangle-|4\rangle),
\end{aligned}
$$

with

$$
\begin{gathered}
O|+1\rangle=|+1\rangle, \quad O|-i\rangle=-i|-i\rangle, \\
O|+i\rangle=i|+i\rangle, \quad O|-1\rangle=-|-1\rangle .
\end{gathered}
$$

Under the other discrete symmetries, these states transform as

$$
\begin{aligned}
& C O^{\prime}|+1\rangle=|+1\rangle, \quad C O^{\prime}|-i\rangle=-i|+i\rangle, \\
& C O^{\prime}|+i\rangle=i|-i\rangle, \quad C O^{\prime}|-1\rangle=-|-1\rangle, \\
& C T_{x}|+1\rangle=|+1\rangle, \quad C T_{x}|-i\rangle=|+i\rangle, \\
& C T_{x}|+i\rangle=|-i\rangle, \quad C T_{x}|-1\rangle=|-1\rangle, \\
& C T_{y}|+1\rangle=|+1\rangle, \quad C T_{y}|-i\rangle=-|+i\rangle, \\
& C T_{y}|+i\rangle=-|-i\rangle, \quad C T_{y}|-1\rangle=|-1\rangle .
\end{aligned}
$$

Besides $| \pm 1\rangle$, we can also construct linear combinations of $| \pm i\rangle$, which are eigenstates of $C T_{x}$ and $C T_{y}$ such that

$$
\begin{aligned}
& C T_{x}|+1\rangle=|+1\rangle, \\
& C T_{x} \frac{1}{\sqrt{2}}(|+i\rangle \pm|-i\rangle)= \pm \frac{1}{\sqrt{2}}(|+i\rangle \pm|-i\rangle), \\
& C T_{x}|-1\rangle=|-1\rangle, \\
& C T_{y}|+1\rangle=|+1\rangle, \\
& C T_{y} \frac{1}{\sqrt{2}}(|+i\rangle \pm|-i\rangle)=\mp \frac{1}{\sqrt{2}}(|+i\rangle \pm|-i\rangle), \\
& C T_{y}|-1\rangle=|-1\rangle .
\end{aligned}
$$

This implies that in the columnar phase, in a finite volume there are four almost degenerate ground states with $\left(C T_{x}, C T_{y}\right)$ quantum numbers $(+,+),(+,-),(-,+),(+,+)$. 


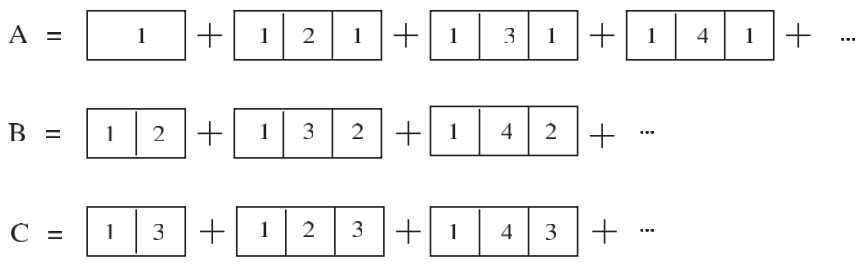

FIG. 8. A pictorial representation of the transition amplitudes appearing in Eq. (3.5). The numbers 1, 2, 3, and 4 represent the four columnar phases.

These four states are eigenstates of a reduced transfer matrix

$$
T=\exp (-\beta H)=\left(\begin{array}{llll}
A & B & C & B \\
B & A & B & C \\
C & B & A & B \\
B & C & B & A
\end{array}\right) .
$$

Here, $A, B$, and $C$ are transition amplitudes connecting the various phases. The corresponding transfer matrix eigenvalues are

$$
\begin{aligned}
& \exp \left(-\beta E_{+1}\right)=A+2 B+C, \\
& \exp \left(-\beta E_{ \pm i}\right)=A-C, \\
& \exp \left(-\beta E_{-1}\right)=A-2 B+C .
\end{aligned}
$$

Notice only three transition amplitudes appear in Eq. (3.5). This is because the transitions from $|+1\rangle$ to $|i\rangle$ and $|+1\rangle$ to $|-i\rangle$ are of the same type. A pictorial representation for each transition amplitude is depicted in Fig. 8. Using a dilute instanton gas approximation, one can derive analytic expressions for the transfer matrix elements $A, B$, and $C$. There are instantons that represent tunneling events between the phases 1 or 3 to 2 or 4 . These instantons have a Boltzmann weight $\delta_{\perp} \exp \left(-\alpha_{\perp} L_{x} L_{y}\right)$. In addition, there are instantons connecting the phases 1 with 3 , as well as 2 with 4 . These have a Boltzmann weight $\delta_{\|} \exp \left(-\alpha_{\|} L_{x} L_{y}\right)$. The factors $\delta_{\perp}$ and $\delta_{\|}$describe capillary wave fluctuations of the instantons. Denoting the free energy in a bulk phase by $f$, an additional Boltzmann factor $\exp \left(-\beta f L_{x} L_{y}\right)$ arises as well. The explicit calculation for $A, B, C$ then yields the following expressions for the exponentially small energy gaps:

$$
\begin{aligned}
& E_{ \pm i}-E_{+1}=2 \delta_{\perp} \exp \left(-\alpha_{\perp} L_{x} L_{y}\right)+2 \delta_{\|} \exp \left(-\alpha_{\|} L_{x} L_{y}\right), \\
& E_{-1}-E_{+1}=4 \delta_{\perp} \exp \left(-\alpha_{\perp} L_{x} L_{y}\right) .
\end{aligned}
$$

In this calculation, we have assumed that the interfaces that correspond to a 1-3 (or 2-4) instanton with an interface tension $\alpha_{\|}$are not completely wet by the other phases 2,4 (or 1,3). This assumption implies $\alpha_{\|}<2 \alpha_{\perp}$. Antonov's rule [34] excludes $\alpha_{\|}>2 \alpha_{\perp}$, because interfaces with tension $\alpha_{\|}$would then be unstable against the decay into two interfaces with tension $\alpha_{\perp}$. This is the situation of complete wetting. Interfaces with tension $\alpha_{\|}$then simply do not exist and the corresponding equations turn into

$$
\begin{aligned}
& E_{ \pm i}-E_{+1}=2 \delta_{\perp} \exp \left(-\alpha_{\perp} L_{x} L_{y}\right), \\
& E_{-1}-E_{+1}=4 \delta_{\perp} \exp \left(-\alpha_{\perp} L_{x} L_{y}\right)=2\left(E_{ \pm i}-E_{+1}\right) .
\end{aligned}
$$

Equidistant level spacings are characteristic for complete wetting. At least for $\lambda \rightarrow-\infty$, one indeed expects complete wetting.

\section{B. Low-energy spectrum in the plaquette phase}

We now consider the finite-volume energy spectrum in the plaquette phase. Similar to the analysis in the columnar phase, we define the four plaquette eigenstates as

$$
\begin{aligned}
|+1\rangle^{\prime} & =\frac{1}{2}(|A\rangle+|B\rangle+|C\rangle+|D\rangle), \\
|-i\rangle^{\prime} & =\frac{1}{2}(|A\rangle+i|B\rangle-|C\rangle-i|D\rangle), \\
|+i\rangle^{\prime} & =\frac{1}{2}(|A\rangle-i|B\rangle-|C\rangle+i|D\rangle), \\
|-1\rangle^{\prime} & =\frac{1}{2}(|A\rangle-|B\rangle+|C\rangle-|D\rangle) .
\end{aligned}
$$

Under the discrete symmetries they transform as

$$
\begin{aligned}
O|+1\rangle^{\prime} & =|+1\rangle^{\prime}, \quad O|-i\rangle^{\prime}=-i|-i\rangle^{\prime}, \\
O|+i\rangle^{\prime} & =i|+i\rangle^{\prime}, \quad O|-1\rangle^{\prime}=-|-1\rangle^{\prime}, \\
C O^{\prime}|+1\rangle^{\prime} & =|+1\rangle^{\prime}, \quad C O^{\prime}|-i\rangle^{\prime}=-|+i\rangle^{\prime}, \\
C O^{\prime}|+i\rangle^{\prime} & =-|-i\rangle^{\prime}, \quad C O^{\prime}|-1\rangle^{\prime}=|-1\rangle^{\prime}, \\
C T_{x}|+1\rangle^{\prime} & =|+1\rangle^{\prime}, \quad C T_{x}|-i\rangle^{\prime}=-i|+i\rangle^{\prime}, \\
C T_{x}|+i\rangle^{\prime} & =i|-i\rangle^{\prime}, \quad C T_{x}|-1\rangle^{\prime}=-|-1\rangle^{\prime}, \\
C T_{y}|+1\rangle^{\prime} & =|+1\rangle^{\prime}, \quad C T_{y}|-i\rangle^{\prime}=i|+i\rangle^{\prime}, \\
C T_{y}|+i\rangle^{\prime} & =-i|-i\rangle^{\prime}, \quad C T_{y}|-1\rangle^{\prime}=-|-1\rangle^{\prime} .
\end{aligned}
$$

Besides $| \pm 1\rangle^{\prime}$, we can also construct linear combinations of $| \pm i\rangle^{\prime}$, which are eigenstates of $C T_{x}$ and $C T_{y}$ such that

$$
\begin{aligned}
& C T_{x}|+1\rangle^{\prime}=|+1\rangle^{\prime}, \\
& C T_{x} \frac{1}{\sqrt{2}}\left(|+i\rangle^{\prime} \pm i|-i\rangle^{\prime}\right)= \pm \frac{1}{\sqrt{2}}\left(|+i\rangle^{\prime} \pm i|-i\rangle^{\prime}\right), \\
& C T_{x}|-1\rangle^{\prime}=-|-1\rangle^{\prime}, \\
& C T_{y}|+1\rangle^{\prime}=|+1\rangle^{\prime}, \\
& C T_{y} \frac{1}{\sqrt{2}}\left(|+i\rangle^{\prime} \pm i|-i\rangle^{\prime}\right)=\mp \frac{1}{\sqrt{2}}\left(|+i\rangle^{\prime} \pm i|-i\rangle^{\prime}\right), \\
& C T_{y}|-1\rangle^{\prime}=-|-1\rangle^{\prime} .
\end{aligned}
$$

Like in the columnar phase, in the plaquette phase there are four almost degenerate ground states. However, in contrast to the columnar phase, their $\left(C T_{x}, C T_{y}\right)$ quantum numbers are $(+,+),(+,-),(-,+)$, and $(-,-)$. In particular, the quantum numbers of the third excited state are different in the two cases. The calculation of the energy spectrum in the plaquette phase is the same as in the columnar phase and shall not be repeated here.

\section{Low-energy spectrum in the mixed phase}

Finally we discuss the lowest states in the finite-volume energy spectrum of the mixed phase. Here we have a total of eight states that become degenerate in the infinite volume limit, with exponentially small gaps at finite volume. Let us construct the states as eigenstates of an explicitly broken and thus only approximate $\mathbb{Z}(8)$ symmetry. Defining $z=\exp (2 \pi i / 8)$, we 
obtain

$$
\begin{aligned}
|+1\rangle^{\prime \prime} & =\frac{1}{\sqrt{8}}\left(\left|A_{1}\right\rangle+\left|A_{2}\right\rangle+\left|B_{2}\right\rangle+\left|B_{3}\right\rangle+\left|C_{3}\right\rangle+\left|C_{4}\right\rangle+\left|D_{4}\right\rangle+\left|D_{1}\right\rangle\right), \\
|z\rangle^{\prime \prime} & =\frac{1}{\sqrt{8}}\left(\left|A_{1}\right\rangle+z\left|A_{2}\right\rangle+i\left|B_{2}\right\rangle+z^{3}\left|B_{3}\right\rangle-\left|C_{3}\right\rangle+z^{5}\left|C_{4}\right\rangle-i\left|D_{4}\right\rangle+z^{7}\left|D_{1}\right\rangle\right), \\
|+i\rangle^{\prime \prime} & =\frac{1}{\sqrt{8}}\left(\left|A_{1}\right\rangle+i\left|A_{2}\right\rangle-\left|B_{2}\right\rangle-i\left|B_{3}\right\rangle+\left|C_{3}\right\rangle+i\left|C_{4}\right\rangle-\left|D_{4}\right\rangle-i\left|D_{1}\right\rangle\right), \\
\left|z^{3}\right\rangle^{\prime \prime} & =\frac{1}{\sqrt{8}}\left(\left|A_{1}\right\rangle+z^{3}\left|A_{2}\right\rangle-i\left|B_{2}\right\rangle+z\left|B_{3}\right\rangle-\left|C_{3}\right\rangle+z^{7}\left|C_{4}\right\rangle+i\left|D_{4}\right\rangle+z^{5}\left|D_{1}\right\rangle\right), \\
|-1\rangle^{\prime \prime} & =\frac{1}{\sqrt{8}}\left(\left|A_{1}\right\rangle-\left|A_{2}\right\rangle+\left|B_{2}\right\rangle-\left|B_{3}\right\rangle+\left|C_{3}\right\rangle-\left|C_{4}\right\rangle+\left|D_{4}\right\rangle-\left|D_{1}\right\rangle\right), \\
\left|z^{5}\right\rangle^{\prime \prime} & =\frac{1}{\sqrt{8}}\left(\left|A_{1}\right\rangle+z^{5}\left|A_{2}\right\rangle+i\left|B_{2}\right\rangle+z^{7}\left|B_{3}\right\rangle-\left|C_{3}\right\rangle+z\left|C_{4}\right\rangle-i\left|D_{4}\right\rangle+z^{3}\left|D_{1}\right\rangle\right), \\
|-i\rangle^{\prime \prime} & =\frac{1}{\sqrt{8}}\left(\left|A_{1}\right\rangle-i\left|A_{2}\right\rangle-\left|B_{2}\right\rangle+i\left|B_{3}\right\rangle+\left|C_{3}\right\rangle-i\left|C_{4}\right\rangle-\left|D_{4}\right\rangle+i\left|D_{1}\right\rangle\right), \\
\left|z^{7}\right\rangle^{\prime \prime} & =\frac{1}{\sqrt{8}}\left(\left|A_{1}\right\rangle+z^{7}\left|A_{2}\right\rangle-i\left|B_{2}\right\rangle+z^{5}\left|B_{3}\right\rangle-\left|C_{3}\right\rangle+z^{3}\left|C_{4}\right\rangle+i\left|D_{4}\right\rangle+z\left|D_{1}\right\rangle\right) .
\end{aligned}
$$

This gives rise to the following transformation rules:

$$
\begin{aligned}
O|+1\rangle^{\prime \prime} & =|+1\rangle^{\prime \prime}, \quad O|z\rangle^{\prime \prime}=-i|z\rangle^{\prime \prime}, \quad O|+i\rangle^{\prime \prime}=-|+i\rangle^{\prime \prime}, \quad O\left|z^{3}\right\rangle^{\prime \prime}=i\left|z^{3}\right\rangle^{\prime \prime}, \\
O|-1\rangle^{\prime \prime} & =|-1\rangle^{\prime \prime}, \quad O\left|z^{5}\right\rangle^{\prime \prime}=-i\left|z^{5}\right\rangle^{\prime \prime}, \quad O|-i\rangle^{\prime \prime}=-|-i\rangle^{\prime \prime}, \quad O\left|z^{7}\right\rangle^{\prime \prime}=i\left|z^{7}\right\rangle^{\prime \prime}, \\
C O^{\prime}|+1\rangle^{\prime \prime} & =|+1\rangle^{\prime \prime}, \quad C O^{\prime}|z\rangle^{\prime \prime}=z^{5}\left|z^{7}\right\rangle^{\prime \prime}, \quad C O^{\prime}|+i\rangle^{\prime \prime}=i|-i\rangle^{\prime \prime}, \quad C O^{\prime}\left|z^{3}\right\rangle^{\prime \prime}=z^{7}\left|z^{5}\right\rangle^{\prime \prime}, \\
C O^{\prime}|-1\rangle^{\prime \prime} & =-1-1\rangle^{\prime \prime}, \quad C O^{\prime}\left|z^{5}\right\rangle^{\prime \prime}=z\left|z^{3}\right\rangle^{\prime \prime}, \quad C O^{\prime}|-i\rangle^{\prime \prime}=-i|+i\rangle^{\prime \prime}, \quad C O^{\prime}\left|z^{7}\right\rangle^{\prime \prime}=z^{3}|z\rangle^{\prime \prime}, \\
C T_{x}|+1\rangle^{\prime \prime} & =|+1\rangle^{\prime \prime}, \quad C T_{x}|z\rangle^{\prime \prime}=z^{7}\left|z^{7}\right\rangle^{\prime \prime}, \quad C T_{x}|+i\rangle^{\prime \prime}=-i|-i\rangle^{\prime \prime}, \quad C T_{x}\left|z^{3}\right\rangle^{\prime \prime}=z^{5}\left|z^{5}\right\rangle^{\prime \prime}, \\
C T_{x}|-1\rangle^{\prime \prime} & =-|-1\rangle^{\prime \prime}, \quad C T_{x}\left|z^{5}\right\rangle^{\prime \prime}=z^{3}\left|z^{3}\right\rangle^{\prime \prime}, \quad C T_{x}|-i\rangle^{\prime \prime}=i|+i\rangle^{\prime \prime}, \quad C T_{x}\left|z^{7}\right\rangle^{\prime \prime}=z|z\rangle^{\prime \prime}, \\
C T_{y}|+1\rangle^{\prime \prime} & =|+1\rangle^{\prime \prime}, \quad C T_{y}|z\rangle^{\prime \prime}=z^{3}\left|z^{7}\right\rangle^{\prime \prime}, \quad C T_{y}|+i\rangle^{\prime \prime}=-i|-i\rangle^{\prime \prime}, \quad C T_{y}\left|z^{3}\right\rangle^{\prime \prime}=z\left|z^{5}\right\rangle^{\prime \prime}, \\
C T_{y}|-1\rangle^{\prime \prime} & =-|-1\rangle^{\prime \prime}, \quad C T_{y}\left|z^{5}\right\rangle^{\prime \prime}=z^{7}\left|z^{3}\right\rangle^{\prime \prime}, \quad C T_{y}|-i\rangle^{\prime \prime}=i|+i\rangle^{\prime \prime}, \quad C T_{y}\left|z^{7}\right\rangle^{\prime \prime}=z^{5}|z\rangle^{\prime \prime} .
\end{aligned}
$$

By construction, the eight states are eigenstates of the approximate continuous $\mathrm{U}(1)$ symmetry $U$ restricted to $\mathbb{Z}(8)$,

$$
\begin{aligned}
& U|+1\rangle^{\prime \prime}=|+1\rangle^{\prime \prime}, \quad U|z\rangle^{\prime \prime}=z|z\rangle^{\prime \prime}, \\
& U|+i\rangle^{\prime \prime}=i|+i\rangle^{\prime \prime}, \quad U\left|z^{3}\right\rangle^{\prime \prime}=z^{3}\left|z^{3}\right\rangle^{\prime \prime}, \\
& U|-1\rangle^{\prime \prime}=-|-1\rangle^{\prime \prime}, \quad U\left|z^{5}\right\rangle^{\prime \prime}=z^{5}\left|z^{5}\right\rangle^{\prime \prime}, \\
& U|-i\rangle^{\prime \prime}=-i|-i\rangle^{\prime \prime}, \quad U\left|z^{7}\right\rangle^{\prime \prime}=z^{7}\left|z^{7}\right\rangle^{\prime \prime} .
\end{aligned}
$$

The symmetries $C T_{x}, C T_{y}$, and $C O^{\prime}$ have $|+1\rangle^{\prime \prime}$ and $|-1\rangle^{\prime \prime}$ unmixed, and they mix $|+i\rangle^{\prime \prime}$ with $|-i\rangle^{\prime \prime},|z\rangle^{\prime \prime}$ with $\left|z^{7}\right\rangle^{\prime \prime}=$ $\left|z^{*}\right\rangle^{\prime \prime}$, and $\left|z^{3}\right\rangle^{\prime \prime}$ with $\left|z^{5}\right\rangle^{\prime \prime}=\left|z^{3 *}\right\rangle^{\prime \prime}$. The energy spectrum will thus contain two nondegenerate states, as well as three pairs of twofold degenerate states. We do not explicitly work out the energy spectrum, but point out that in a mixed phase eight finite-volume states become degenerate in the infinite volume limit, while for the columnar or plaquette phase only four states become degenerate.

\section{LOW-ENERGY EFFECTIVE THEORY}

In Ref. [19], we found strong numerical evidence for an emergent soft pseudo-Goldstone mode at and below the RK point. In this section, we discuss the theoretical concepts underlying our numerical analysis that we present in the next section. These include the effective field description of the pseudo-Goldstone boson mode and the energy spectrum in a finite volume. It should be pointed out that the content of Sec. IV A overlaps with relevant material discussed in Ref. [19]. For completeness and for the benefit of the readers, these discussions are included in Sec. IV A of the current manuscript.

\section{A. Goldstone boson fields, symmetries, Lagrangian}

The basic degree of freedom in the effective theory-the soft pseudo-Goldstone mode-is parametrized by the angle $\varphi=\frac{1}{2}\left(\varphi_{1}+\varphi_{2}+\frac{\pi}{4}\right)$. Note that the angles $\varphi_{1}$ and $\varphi_{2}$ have been defined in Eq. (2.13). Under the various symmetries, the angle $\varphi$ transforms as

$$
\begin{aligned}
& { }^{C T_{x}} \varphi=\pi-\varphi, \quad{ }^{C T_{y}} \varphi=\frac{\pi}{2}-\varphi, \\
& { }^{o} \varphi=\frac{\pi}{4}+\varphi, \quad{ }^{C O^{\prime}} \varphi=-\frac{\pi}{4}-\varphi .
\end{aligned}
$$


The leading Euclidean effective Lagrangian takes the form

$$
\mathcal{L}=\frac{\rho}{2}\left(\frac{1}{c^{2}} \partial_{t} \varphi \partial_{t} \varphi+\partial_{i} \varphi \partial_{i} \varphi\right)+\kappa\left(\partial_{i} \partial_{i} \varphi\right)^{2}+\delta \cos ^{2}(4 \varphi),
$$

which is identical with the effective Lagrangian of the $(2+1)$ dimensional $\mathbb{R} P(1)$ model. Note that the angles $\varphi$ and $\varphi+\pi$ are indistinguishable, such that the physical Hilbert space only contains states that are invariant under this shift.

While $\rho$ is the spin stiffness, the quantity $c$ is the limiting velocity of the pseudo-Goldstone boson. The term proportional to the low-energy effective constant $\delta$ explicitly breaks the emergent $\mathrm{SO}(2)$ symmetry to the discrete subgroup $\mathbb{Z}(8)$. Accordingly, we are dealing with a light pseudo-Goldstone mode with mass

$$
M c=4 \sqrt{2|\delta| / \rho} .
$$

At the RK point $(\lambda=1)$, all flux configurations cost zero energy in their ground state. This implies that the individual effective couplings $\delta$ and $\rho$ (but not the ratio $\rho / c^{2}$ ) are zero. Note that the condition $\rho=0$ at the RK point is an analytic result which does not require any fine-tuning. In this case, the quartic kinetic term proportional to $\kappa$ becomes the dominant contribution. Remarkably, $\partial_{i} \partial_{i} \varphi=0$ for all configurations of static external charges in their ground state, such that this term indeed does not contribute any ground state energy. This is not true for the term $\sum_{i=1,2} \partial_{i} \partial_{i} \varphi \partial_{i} \partial_{i} \varphi$. Hence, such a term cannot arise at the RK point, but it can arise away from it. Also all terms in the potential energy vanish at the RK point.

\section{B. Rotor spectrum}

Let us first consider the spectrum of vacuum states in a periodic volume $L_{1} \times L_{2}$ at $\delta=0$. At zero temperature, to lowest order, we may assume $\varphi(x, t)=\varphi(t)$, i.e., the lowenergy dynamics reduces to the one of the spatial zero-mode, which represents a single quantum mechanical degree of freedom. The action then reduces to

$$
S[\varphi]=\int d t\left[\frac{\rho L_{1} L_{2}}{2 c^{2}} \partial_{t} \varphi \partial_{t} \varphi+\delta L_{1} L_{2} \cos ^{2}(4 \varphi)\right],
$$

and the corresponding quantum mechanical Hamilton operator is given by

$$
H_{\mathrm{eff}}=-\frac{c^{2}}{2 \rho L_{1} L_{2}} \partial_{\varphi}^{2}+\delta L_{1} L_{2} \cos ^{2}(4 \varphi) .
$$

At $\delta=0$, this describes a free "particle" on a circle. The corresponding energy eigenstates and eigenvalues are

$$
\psi_{m}(\varphi)=\frac{1}{\sqrt{2 \pi}} \exp (\operatorname{im} \varphi), \quad E_{m}=\frac{m^{2} c^{2}}{2 \rho L_{1} L_{2}} .
$$

Since $\varphi$ and $\varphi+\pi$ are physically equivalent, $m$ is restricted to even integers.

Let us consider the effects of small $\delta$ in perturbation theory. The ground state with $m=0$ is nondegenerate and has a constant wave function

$$
\psi_{0}(\varphi)=\frac{1}{\sqrt{2 \pi}} .
$$

Its energy shift is

$$
E_{0}^{(1)}=\delta L_{1} L_{2}\left\langle\psi_{0}\left|\cos ^{2}(4 \varphi)\right| \psi_{0}\right\rangle=\frac{\delta L_{1} L_{2}}{2} .
$$

The excited states with $m= \pm 2, \pm 4, \ldots$ are twofold degenerate. Their energy shifts result from

$$
\begin{aligned}
V_{m, m}= & V_{-m,-m}=\frac{\delta L_{1} L_{2}}{2}, \\
V_{m,-m}= & V_{-m, m}=\frac{\delta L_{1} L_{2}}{16 \pi}\left[\frac{\sin (2(m-4) \varphi)}{m-4}+\frac{2 \sin (2 m \varphi)}{m}\right. \\
& \left.+\frac{\sin (2(m+4) \varphi)}{m+4}\right]_{0}^{2 \pi} .
\end{aligned}
$$

The case $m= \pm 4$ thus needs to be considered separately. Since

$$
\lim _{m \rightarrow \pm 4} V_{m,-m}=\frac{\delta L_{1} L_{2}}{4},
$$

the corresponding energy shift takes the form

$$
E_{4 \pm}^{(1)}=\frac{\delta L_{1} L_{2}}{2} \pm \frac{\delta L_{1} L_{2}}{4},
$$

and the previously degenerate energy levels split,

$$
E_{ \pm 4}-E_{0}=\frac{8 c^{2}}{\rho L_{1} L_{2}} \pm \frac{\delta L_{1} L_{2}}{4} .
$$

This formula is only valid in the regime

$$
\delta L_{1} L_{2} \ll \frac{c^{2}}{\rho L_{1} L_{2}} .
$$

For $m= \pm 2, \pm 6, \pm 8, \ldots$, there is no such effect and we simply have

$$
E_{ \pm m}-E_{0}=\frac{m^{2} c^{2}}{2 \rho L_{1} L_{2}} .
$$

We now turn to second-order perturbation theory in $\delta$. Note that the leading-order correction to the higher excited states $m= \pm 2, \pm 4, \ldots$ only arises at order $\delta^{2}$. To avoid degenerate perturbation theory, we separately consider even and odd wave functions. We begin with the even wave functions

$\psi_{m}^{e}(\varphi)=\frac{1}{\sqrt{\pi}} \cos (m \varphi), \quad E_{m}=\frac{m^{2} c^{2}}{2 \rho L_{1} L_{2}}, \quad \psi_{0}^{e}(\varphi)=\frac{1}{\sqrt{2 \pi}}$.

With the matrix elements, $m, n>0$,

$$
\langle m|V(\varphi)| n\rangle=\frac{\delta L_{1} L_{2}}{\pi} \int_{0}^{2 \pi} d \varphi \cos (m \varphi) \cos ^{2}(4 \varphi) \cos (n \varphi),
$$

we obtain

$$
\begin{aligned}
E_{0}^{\mathrm{even},(2)} & =-\sum_{n \neq 0} \frac{|\langle 0|V(\varphi)| n\rangle|^{2}}{E_{n}-E_{0}} \\
& =-\frac{|\langle 0|V(\varphi)| 8\rangle|^{2}}{E_{8}-E_{0}}=-\frac{\delta^{2} \rho L_{1}^{3} L_{2}^{3}}{256 c^{2}},
\end{aligned}
$$




$$
\begin{aligned}
E_{2}^{\mathrm{even},(2)} & =-\sum_{n \neq 2} \frac{|\langle 2|V(\varphi)| n\rangle|^{2}}{E_{n}-E_{2}} \\
& =-\frac{|\langle 2|V(\varphi)| 6\rangle|^{2}}{E_{6}-E_{2}}-\frac{|\langle 2|V(\varphi)| 10\rangle|^{2}}{E_{10}-E_{2}}=-\frac{\delta^{2} \rho L_{1}^{3} L_{2}^{3}}{192 c^{2}}, \\
E_{4}^{\mathrm{even},(2)} & =-\sum_{n \neq 4} \frac{|\langle 4|V(\varphi)| n\rangle|^{2}}{E_{n}-E_{4}} \\
& =-\frac{|\langle 4|V(\varphi)| 12\rangle|^{2}}{E_{12}-E_{4}}=-\frac{\delta^{2} \rho L_{1}^{3} L_{2}^{3}}{1024 c^{2}} .
\end{aligned}
$$

Analogously, for the odd wave functions $(m, n>0)$,

$$
\psi_{m}^{o}(\varphi)=\frac{1}{\sqrt{\pi}} \sin (m \varphi), \quad E_{m}=\frac{m^{2} c^{2}}{2 \rho L_{1} L_{2}},
$$

and with

$$
\langle m|V(\varphi)| n\rangle=\frac{\delta L_{1} L_{2}}{\pi} \int_{0}^{2 \pi} d \varphi \sin (m \varphi) \cos ^{2}(4 \varphi) \sin (n \varphi),
$$

second-order perturbation theory leads to

$$
\begin{aligned}
E_{2}^{\text {odd,(2) }} & =-\sum_{n \neq 2} \frac{|\langle 2|V(\varphi)| n\rangle|^{2}}{E_{n}-E_{2}} \\
& =-\frac{|\langle 2|V(\varphi)| 6\rangle|^{2}}{E_{6}-E_{2}}-\frac{|\langle 2|V(\varphi)| 10\rangle|^{2}}{E_{10}-E_{2}}=-\frac{\delta^{2} \rho L_{1}^{3} L_{2}^{3}}{192 c^{2}} \\
E_{4}^{\text {odd, (2) }} & =-\sum_{n \neq 4} \frac{|\langle 4|V(\varphi)| n\rangle|^{2}}{E_{n}-E_{4}} \\
& =-\frac{|\langle 4|V(\varphi)| 12\rangle|^{2}}{E_{12}-E_{4}}=-\frac{\delta^{2} \rho L_{1}^{3} L_{2}^{3}}{1024 c^{2}} .
\end{aligned}
$$

We now proceed with a nonperturbative treatment of $\delta$ and consider the nonperturbative Schrödinger equation that takes the form of a Hill equation:

$$
\begin{aligned}
& -\frac{1}{2} \delta_{\varphi}^{2} \psi(\varphi)+V_{0} \cos ^{2}(4 \varphi) \psi(\varphi)=\varepsilon \psi(\varphi), \\
& V_{0}=\frac{\delta \rho L_{1}^{2} L_{2}^{2}}{c^{2}}, \quad \varepsilon=\frac{\rho L_{1} L_{2}}{c^{2}} E .
\end{aligned}
$$

Since $\varphi$ and $\varphi+\pi$ are to be identified, we introduce a new angle as $\varphi^{\prime}=4 \varphi$, with $\varphi^{\prime}$ having period $4 \pi$. The above equation is then converted into the following Mathieu equation:

$$
-\delta_{\varphi^{\prime}}^{2} \psi\left(\varphi^{\prime}\right)+\frac{V_{0}}{16} \cos \left(2 \varphi^{\prime}\right) \psi\left(\varphi^{\prime}\right)=\left(\frac{\varepsilon}{8}-\frac{V_{0}}{16}\right) \psi\left(\varphi^{\prime}\right) .
$$

The corresponding solutions are even and odd Mathieu functions

$$
\psi_{2 m}\left(\varphi^{\prime}\right)=\frac{1}{\sqrt{\pi}} c e_{m}\left(\varphi^{\prime}\right), \quad \psi_{2 m+1}\left(\varphi^{\prime}\right)=\frac{1}{\sqrt{\pi}} s e_{m}\left(\varphi^{\prime}\right),
$$

with eigenvalue $\lambda_{0}$ given to lowest order by

$$
\lambda_{0}=\frac{\varepsilon_{0}}{8}-\frac{V_{0}}{16}=-\frac{1}{2}\left(\frac{V_{0}}{32}\right)^{2}+\mathcal{O}\left(V_{0}^{4}\right) .
$$

Accordingly, the ground-state energy reads

$$
E_{0}=\frac{\delta L_{1} L_{2}}{2}-\frac{\delta^{2} \rho L_{1}^{3} L_{2}^{3}}{256 c^{2}}+\mathcal{O}\left(\delta^{4}\right),
$$

in agreement with the leading perturbative results. In the nonperturbative regime, for the excited states, we obtain

$$
E_{m}=\frac{c^{2}}{\rho L_{1} L_{2}} \varepsilon_{m}=\frac{\delta L_{1} L_{2}}{2}+\frac{8 c^{2}}{\rho L_{1} L_{2}} \lambda_{m}\left(\frac{\delta \rho L_{1}^{2} L_{2}^{2}}{16 c^{2}}\right) .
$$

The energy splittings in the rotor spectrum are thus given by the eigenvalues

$$
\lambda_{m}=\lambda_{m}\left(\frac{\delta \rho L_{1}^{2} L_{2}^{2}}{16 c^{2}}\right)
$$

of the Mathieu equation

$$
E_{m}-E_{0}=\frac{8 c^{2}}{\rho L_{1} L_{2}}\left[\lambda_{m}\left(\frac{\delta \rho L_{1}^{2} L_{2}^{2}}{16 c^{2}}\right)-\lambda_{0}\left(\frac{\delta \rho L_{1}^{2} L_{2}^{2}}{16 c^{2}}\right)\right] .
$$

As a consistency check we also consider the eigenvalue $\lambda_{1}$ that corresponds to the odd Mathieu function $s e_{1}\left(\varphi^{\prime}\right)$,

$$
\begin{aligned}
\lambda_{1} & =\frac{\varepsilon_{1}}{8}-\frac{V_{0}}{16} \\
& =1-\frac{V_{0}}{32}-\frac{1}{8}\left(\frac{V_{0}}{32}\right)^{2}+\frac{1}{64}\left(\frac{V_{0}}{32}\right)^{3}+\mathcal{O}\left(V_{0}^{4}\right) .
\end{aligned}
$$

Accordingly, the energy $E_{1}$ is given by

$$
E_{1}=\frac{8 c^{2}}{\rho L_{1} L_{2}}+\frac{\delta L_{1} L_{2}}{4}-\frac{\delta^{2} \rho L_{1}^{3} L_{2}^{3}}{1024 c^{2}}+\frac{\delta^{3} \rho^{2} L_{1}^{5} L_{2}^{5}}{262144 c^{4}}+\mathcal{O}\left(\delta^{4}\right),
$$

such that

$$
\begin{aligned}
E_{1}-E_{0}= & \frac{8 c^{2}}{\rho L_{1} L_{2}}-\frac{\delta L_{1} L_{2}}{4}+\frac{3 \delta^{2} \rho L_{1}^{3} L_{2}^{3}}{1024 c^{2}} \\
& +\frac{\delta^{3} \rho^{2} L_{1}^{5} L_{2}^{5}}{262144 c^{4}}+\mathcal{O}\left(\delta^{4}\right) .
\end{aligned}
$$

This is consistent with the leading-order perturbative calculation when one identifies the state corresponding to $s e_{1}\left(\varphi^{\prime}\right)$ with the state $m=-4$. Note that the results of secondorder perturbation theory in $\delta$ are also consistent with the expansion of the Mathieu function eigenvalues. The theoretical results derived in this section will be compared with exact diagonalization and Monte Carlo simulation results in Secs. V and VI, respectively.

\section{EXACT DIAGONALIZATION RESULTS}

In this section, we discuss exact diagonalization results for $L_{1} \times L_{2}$ lattices with $L_{1}, L_{2} \in\{4,6,8\}$, which allow us to determine some low-energy parameters of the effective field theory discussed in the previous section. Part of the spectrum 


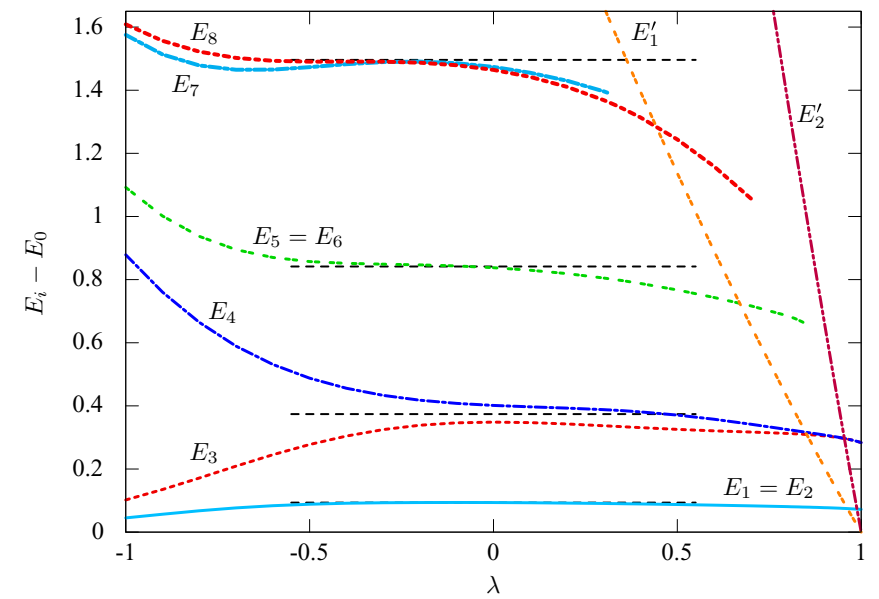

FIG. 9. Energy spectrum on an $8^{2}$ lattice as a function of the RK coupling $\lambda$. This figure is reproduced from Ref. [19] for the reader's benefit.

analysis has already appeared in Ref. [19]. The inclusion of these results is for the benefit of the readers.

Using exact diagonalization we were able to calculate the low-lying energy spectrum on lattices up to $8 \times 8$. Figure 9 shows the energy gaps on the largest lattice. For $\lambda<1$, the ground state is nondegenerate and transforms trivially under the symmetry operations, i.e., it has quantum numbers $\left(C T_{x}, C T_{y}\right)=(+,+)$. The first two excited states with energy gap $E_{1}=E_{2}$ are degenerate and have quantum numbers $(+,-)$ and $(-,+)$, while the next excited state with energy gap $E_{3}$ has again quantum numbers $(+,+)$. As Fig. 10 shows for $\lambda=-1$, the energy gaps of these three excited states decrease exponentially with the volume $L_{1} L_{2}$, i.e., $E_{1,2}, E_{3} \sim \exp \left(-\alpha L_{1} L_{2}\right)$ for $-0.2 \lesssim \lambda \lesssim 0.8$. The fact that the gap between the finite-volume ground state and the three first excited states is exponentially small indicates that four phases coexist at zero temperature. The $\left(C T_{x}, C T_{y}\right)$ quantum numbers $(+,+),(+,-),(-,+),(+,+)$ indicate that we are in a columnar and not in a plaquette phase.

If the columnar phase were replaced by the plaquette phase for larger values of $\lambda$, one would expect a level crossing of the excited $(+,+)$ state with the lowest $(-,-)$ state. Interestingly,

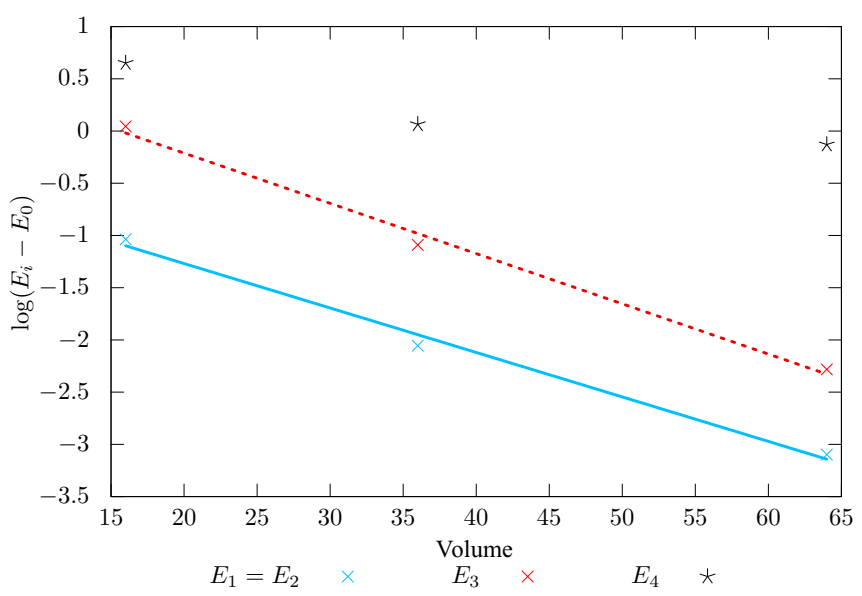

FIG. 10. Logarithmic energy gaps as a function of the volume at $\lambda=-1$. no such level crossing arises in our exact diagonalization study. Notably, the next excited state with energy gap $E_{4}$, does not decrease exponentially with the volume. It has quantum numbers $(-,-)$ and almost degenerates with the $(+,+)$ state with energy $E_{3}$ for $-0.2 \lesssim \lambda \lesssim 0.8$. Furthermore, the next two states with energy $E_{5}=E_{6}$ are exactly degenerate and again have quantum numbers $(+,-)$ and $(-,+)$. The next states, with energies $E_{7}$ and $E_{8}$ are once more almost degenerate and transform as $(+,+)$ and $(-,-)$. The energy ratios of these states are given by $E_{1,2}: E_{3,4}: E_{5,6}: E_{7,8} \approx 1: 4: 9: 16$, which is indicated by the dashed lines in Fig. 9. This hints at an approximate rotor spectrum. Indeed, in Ref. [19], we presented numerical evidence for an emergent approximate spontaneously broken $\mathrm{SO}(2)$ symmetry with an associated pseudo-Goldstone boson. Since the Goldstone boson has a small mass, it does not qualify as a dual photon and the theory remains confining before one reaches the RK point. While the exact diagonalization study alone is not sufficient to come to this conclusion, it is fully consistent with it. We would like to point out that our Fig. 9 compares favorably with Fig. 2 (left) of Ref. [18] for the lower gaps. However, other observables considered in our ED analysis are different from that studied in Ref. [18]. For example, while we extract the low-energy constant $\delta$ and $\rho / c^{2}$ (which will be shown in detail shortly), in Ref. [18], the order parameters are calculated.

Using the analytic results of the effective theory obtained in the previous section, we now estimate some low-energy parameters by comparison with the exact diagonalization results for the rotor spectrum. Figure 11 shows the results for the symmetry breaking parameter $\delta$ (top) and the combination $\frac{c^{2}}{\rho}$ (bottom). These results have been obtained from a global fit using data from $6 \times 6$ and $8 \times 8$ lattices for different values of $\lambda$. Note that $\frac{c^{2}}{\rho}$ is positive for all values of $\lambda$, while $\delta \geqslant 0$ approaches zero near the RK point. Remarkably, the fit works rather well up to values of $\lambda \approx 0.6$. Even though the errors are increasing near the RK point, the results are still consistent with positive values of $\delta$, thus indicating the absence of a phase transition before the RK point. This suggests that the columnar phase extends all the way up to $\lambda=1$. However, the precision reachable with the moderate volumes accessible to exact diagonalization is not sufficient to definitively settle this issue. In Ref. [19], we have provided numerical evidence based on Monte Carlo data obtained on much larger systems, which implies that $\delta$ remains positive until one reaches the RK point, thus excluding a transition into the plaquette phase. Our result is not inconsistent with that of Ref. [18] because Ref. [18] is inconclusive regarding the existence of a phase transition as well.

One may wonder whether a mixed phase [18], sharing features of both the columnar and the plaquette phase, would give rise to a similar finite-volume spectrum. As we have pointed out in the previous section, in the mixed phase eight vacuum states, separated by exponentially small energy gaps, are almost degenerate in a finite volume. This is qualitatively different from the rotor spectrum observed in our exact diagonalization studies. First of all, the energy of the rotor states decreases inversely proportional to and not exponentially with the volume. In addition, on the moderate volumes accessible to exact diagonalization, the observed spectrum 

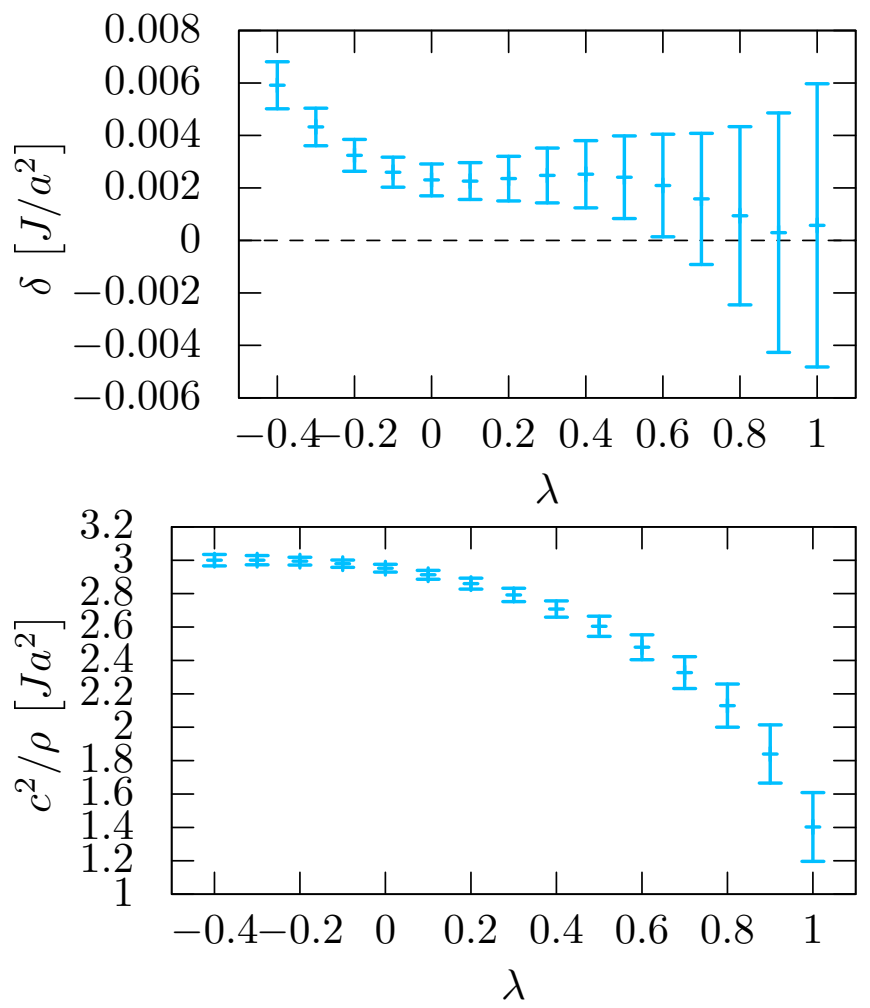

FIG. 11. Results for the parameter $\delta$ (top) and the combination $\frac{c^{2}}{\rho}$ (bottom) of the effective theory from a fit to the exact diagonalization data for different $\lambda$ for the lattice sizes $6 \times 6$ and $8 \times 8$. The error bar that appears in the figures for each value of $\lambda$ is the uncertainty of the calculated quantity from the fit.

contains at least nine rotor states, while the mixed phase would be characterized by eight low-energy states separated from the rest of the spectrum by a gap. Although based on our numerical results, the debated plaquette or mixed phases are not stabilized in the present QDM, these phases may be realized in generalized QDMs more relevant for true

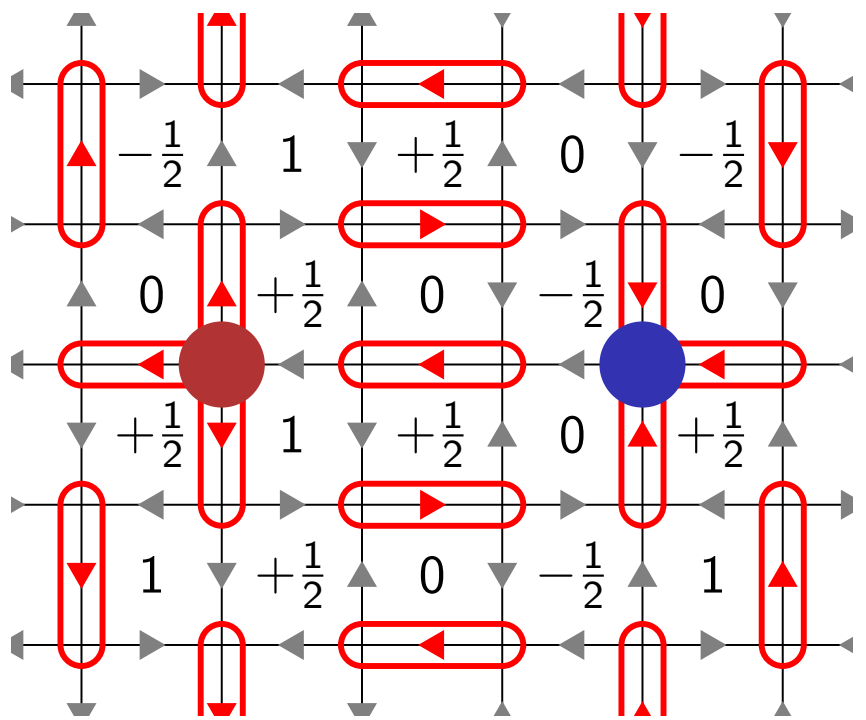

FIG. 12. The presence of two external static charges violates the dimer covering constraint. This figure is reproduced from [19] for the benefit of the readers. quantum spin models [33]. We leave the investigation of these generalized QDMs for future potential studies.

Finally, Fig. 9 shows two sets of states with energies $E_{1}^{\prime}$ and $E_{2}^{\prime}$. These states have the quantum numbers $\left(C T_{x}, C T_{y}\right)=$ $(+,+)$, and represent strings of nonzero electric flux

$$
\mathcal{E}_{i}=\frac{1}{L_{i}} \sum_{x} E_{x, x+\hat{i}}
$$

wrapping around the periodic spatial volume. There are four states with energy $E_{1}^{\prime}$ with electric fluxes $\left(\mathcal{E}_{1}, \mathcal{E}_{2}\right)=$
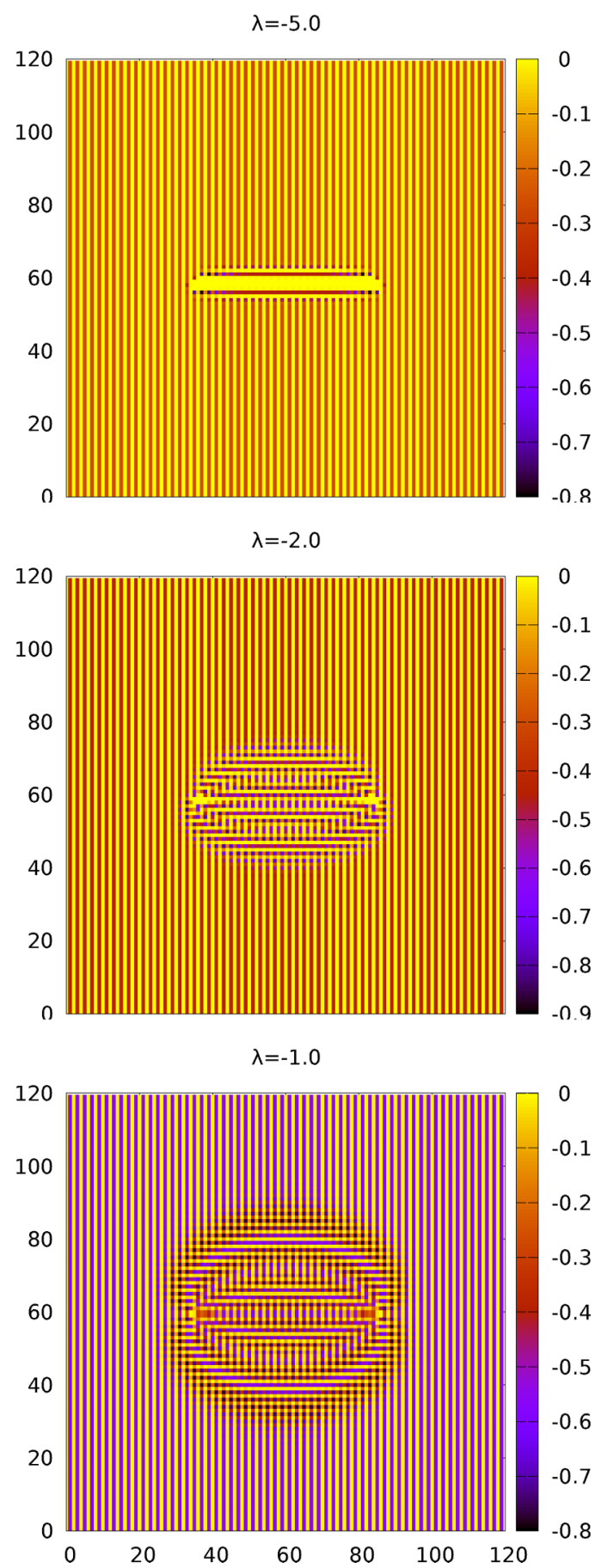

FIG. 13. (Top to bottom) Energy density $-J\left\langle U_{\square}+U_{\square}^{\dagger}\right\rangle$ on a $120 \times 120$ lattice in the presence of two charges \pm 2 , separated by 49 lattice spacings for $\beta J=64$ and $\lambda=-5,-2,-1$. 
$( \pm 1,0),(0, \pm 1)$, while there are two states with energy $E_{2}^{\prime}$ with electric fluxes $( \pm 2,0),(0, \pm 2)$. The energy gaps of these states vanish at the RK point $\lambda=1$. This implies that at this point, flux strings cost zero energy thus signaling deconfinement and the spontaneous breakdown of the $\mathrm{U}(1)^{2}$ center symmetry.

\section{MONTE CARLO RESULTS}

Green's function Monte Carlo simulations have been applied earlier to the square lattice quantum dimer model [16-18], with lattice sizes $L^{2}$ up to $L=48$. In our previous study [19] we have used a more efficient Monte Carlo algorithm that enabled us to reach volumes up to $L^{2}=144 \times 144$ and temperatures down to $T=J / 500$. Our algorithm is based on the height variable representation of the quantum dimer model. More details about the algorithm have been presented in Ref. [19].

Some Monte Carlo data, in particular those which provide convincing numerical evidence that the columnar phase is realized in the square lattice quantum dimer model all the way to the RK point, are already shown in Ref. [19]. Here we present new results and we give a more detailed explanation of the results obtained earlier.

By putting two external static charges \pm 2 (relative to the staggered charge background) into the system, one violates the dimer covering constraint. As depicted in Fig. 12, this leads to two defects, associated with three dimers that overlap at the same lattice point. The two static charges, separated by an odd number of lattice spacings, are connected by an electric flux string and are thus confined. In addition, the flux string fractionalizes into eight individual strands-displaying the four plaquette phases-which each carry electric flux $\frac{1}{4}$, thus adding up to the total flux 2 . The energy density $-J\left\langle U_{\square}+U_{\square}^{\dagger}\right\rangle$ for $\lambda=-5,-2,-1$ is shown in Fig. 13: one notices that, as one moves from large negative values of $\lambda$ towards $\lambda \approx 0$, the individual strands emerge around $\lambda \approx-2$. Furthermore, inside the different strands plaquette order is present. These regions of plaquette order are interfaces separating the various columnar phases. In fact, Fig. 14 implies

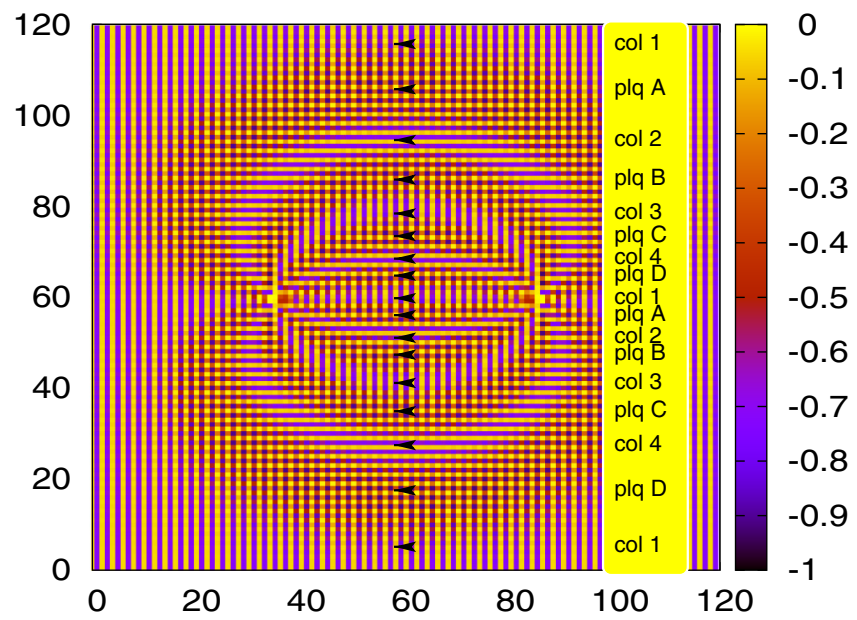

FIG. 14. The appearance of plaquette order, which results from the interface between different columnar phases, in the eight strands. The result is obtained on a $120 \times 120$ lattice with $\beta J=64$ and $\lambda=-0.5$.

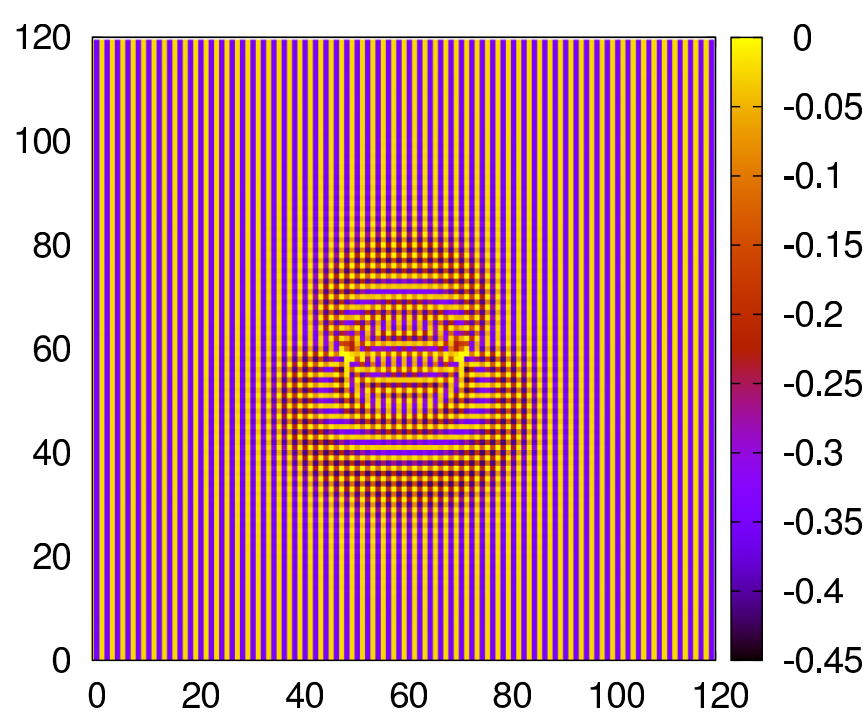

FIG. 15. Energy density $-J\left\langle U_{\square}+U_{\square}^{\dagger}\right\rangle$ on a $120 \times 120$ lattice in the presence of two charges \pm 2 , separated by 43 lattice spacings for $\beta J=64$ and $\lambda=-0.5$.

that as one moves from bottom to top, each of the four possible columnar phases is visited once. The same is true for the four degenerate plaquette orders.

In the presence of two external charges \pm 2 separated along the $x$-axis, both translation and rotation invariance are explicitly broken, while the reflection on the $x$-axis remains an exact symmetry. As a result, one of the columnar phases, with the columns oriented in the $y$ direction, is energetically favored. Interestingly, Fig. 15 shows that for $\lambda=-0.5$, an asymmetric distribution of the eight strands is observed, thus indicating the spontaneous breakdown of the reflection symmetry. Strictly speaking, in an infinite volume spontaneous breaking of the reflection symmetry only arises when the distance between the charges also approaches infinity. At finite distances, the two asymmetric flux patterns, which are related to one another by reflection, coexist with each other through

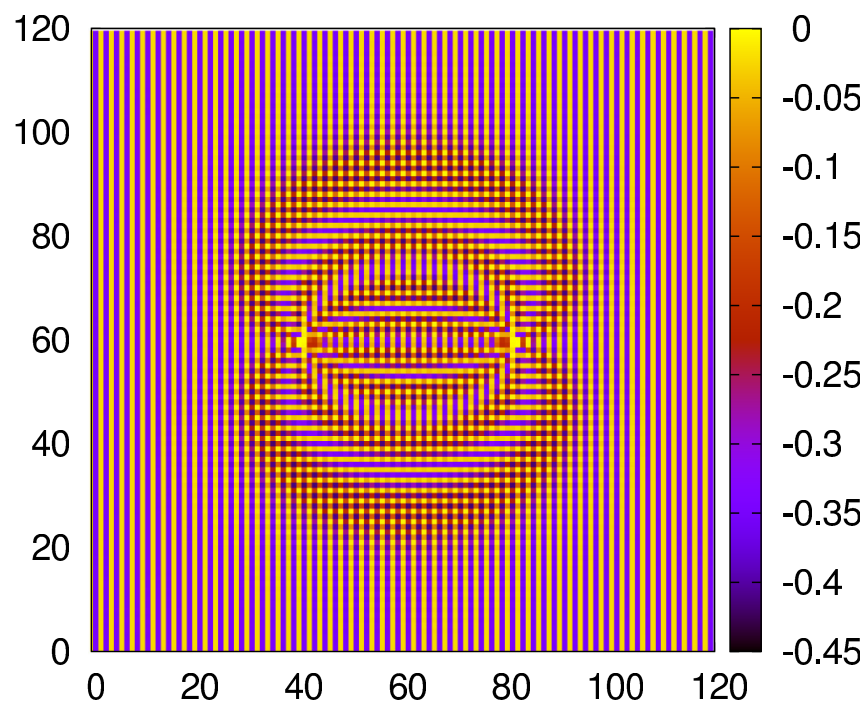

FIG. 16. Energy density $-J\left\langle U_{\square}+U_{\square}^{\dagger}\right\rangle$ on a $120 \times 120$ lattice in the presence of two charges \pm 2 , separated by 53 lattice spacings for $\beta J=64$ and $\lambda=-0.5$. 
quantum tunneling. As the charges are separated further and further, still assuming an infinite volume, tunneling is exponentially suppressed. When we consider a finite volume, the asymmetry in the flux distribution disappears when the distance between the charges becomes compatible with the lattice size (Fig. 16). Squeezing the flux distribution into a small volume leads to a restoration of the spontaneously broken reflection symmetry due to finite-size effects. This scenario also arises for other negative values of $\lambda$.

\section{CONCLUSIONS}

We have investigated the finite-volume energy spectrum of the square lattice quantum dimer model using both exact diagonalization and quantum Monte Carlo. By comparison with analytic predictions we have consolidated our previous evidence that the columnar phase extends all the way to the RK point, without any intervening plaquette or mixed phases. In addition, we have studied a soft pseudo-Goldstone mode that becomes massless at the RK point but still dominates a large region in parameter space away from it. This mode is described by a systematic low-energy effective field theory whose parameters we have extracted by comparison of numerical data with analytic predictions of the effective theory. It will be an interesting topic for future studies to investigate the possible role of the soft mode for the preformation of pairs in the pseudogap regime of high-temperature superconductors. This could be done in the context of hole-doped quantum dimer models [15,35-41].

We have also studied the internal structure of the strings connecting external charges embedded in the confining columnar phase. For topological reasons, the string fractionalizes into strands, each carrying electric flux $\frac{1}{4}$. The flux strands play the role of interfaces separating the four realizations of the columnar phase. As we noted earlier [19], the interior of the flux strands shows plaquette order, despite the fact that the plaquette phase is not stable in the bulk. The interfaces that separate two columnar phases 1 and 3 or 2 and 4, with the columns oriented in the same direction, show the universal phenomenon of complete wetting. This manifests itself by the appearance of a third columnar phase at the interface, with its columns oriented in an orthogonal direction. Hence a 1-3 interface splits into two 1-2-3 or 1-4-3 interfaces. Remarkably, reflections on the lattice axis connecting charges \pm 2 are spontaneously broken, which gives rise to asymmetric electric flux profiles.

As we have seen, the simple square lattice quantum dimer model has a rich confining dynamics, characterized by strings with an intriguing anatomy. Understanding these dynamics required the interplay between numerical simulations and analytic effective field theory calculations. It will be interesting and promising to apply this strategy to quantum dimer models with other lattice geometries $[42,43]$.

\section{ACKNOWLEDGMENTS}

D.B. acknowledges interesting discussions with A. Läuchli. C.P.H. thanks the members of the ITP at Bern University for their hospitality, and acknowledges support through the project Redes Temáticas de Colaboración Académica 2013, UCOL-CA-56. The research leading to these results has received funding from the Schweizerischer Nationalfonds and from the European Research Council under the European Union's Seventh Framework Programme (FP7/2007-2013)/ ERC Grant Agreement No. 339220.

\section{APPENDIX: SYMMETRIES AND CANDIDATE PHASES}

It is important to know how the four order parameters transform under the symmetries $C T_{x}, C T_{y}, O, C O^{\prime}, R_{x}$, and $R_{y}$ of the square lattice quantum dimer model. This is illustrated in Table I.

In Tables II-IV, we show how the different symmetries $C T_{x}, C T_{y}, O, C O^{\prime}, R_{x}$, and $R_{y}$ act on the columnar, plaquette, and mixed phases, respectively.

TABLE I. Transformation properties of the order parameters $M_{i j}$ under the symmetries $S=C T_{x}, C T_{y}, O, C O^{\prime}, R_{x}$, and $R_{y}$. The order parameter $M_{i j}\left[{ }^{S} \mathcal{C}\right]$ evaluated in the transformed configuration ${ }^{S} \mathcal{C}$ as a function of the order parameters $M_{i j}[\mathcal{C}]$ evaluated in the original configuration $\mathcal{C}$.

\begin{tabular}{lcccccc}
\hline \hline$S$ & $C T_{x}$ & $C T_{y}$ & $O$ & $C O^{\prime}$ & $R_{x}$ & $R_{y}$ \\
\hline$M_{11}\left[{ }^{S} \mathcal{C}\right]$ & $-M_{11}[\mathcal{C}]$ & $-M_{22}[\mathcal{C}]$ & $M_{21}[\mathcal{C}]$ & $-M_{21}[\mathcal{C}]$ & $-M_{22}[\mathcal{C}]$ & $-M_{11}[\mathcal{C}]$ \\
$M_{12}\left[{ }^{S} \mathcal{C}\right]$ & $M_{21}[\mathcal{C}]$ & $-M_{12}[\mathcal{C}]$ & $-M_{11}[\mathcal{C}]$ & $-M_{22}[\mathcal{C}]$ & $-M_{12}[\mathcal{C}]$ & $M_{21}[\mathcal{C}]$ \\
$M_{21}\left[{ }^{S} \mathcal{C}\right]$ & $M_{12}[\mathcal{C}]$ & $M_{21}[\mathcal{C}]$ & $-M_{22}[\mathcal{C}]$ & $-M_{11}[\mathcal{C}]$ & $M_{21}[\mathcal{C}]$ & $M_{12}[\mathcal{C}]$ \\
$M_{22}\left[{ }^{S} \mathcal{C}\right]$ & $M_{22}[\mathcal{C}]$ & $-M_{11}[\mathcal{C}]$ & $-M_{12}[\mathcal{C}]$ & $-M_{12}[\mathcal{C}]$ & $-M_{11}[\mathcal{C}]$ & $M_{22}[\mathcal{C}]$ \\
\hline \hline
\end{tabular}

TABLE II. Transformation properties of the four columnar phases 1, 2, 3, 4 under the symmetries $S=C T_{x}, C T_{y}, O, C O^{\prime}, R_{x}$, and $R_{y}$.

\begin{tabular}{lcccccc}
\hline \hline$S$ & $C T_{x}$ & $C T_{y}$ & $O$ & $C O^{\prime}$ & $R_{x}$ & $R_{y}$ \\
\hline$s_{1}$ & 1 & 3 & 2 & 4 & 3 & 1 \\
$s_{2}$ & 4 & 2 & 3 & 3 & 2 & 4 \\
$s_{3}$ & 3 & 1 & 4 & 2 & 1 & 3 \\
$s_{4}$ & 2 & 4 & 1 & 1 & 4 & 2 \\
\hline \hline
\end{tabular}

TABLE III. Transformation properties of the four plaquette phases A, B, C, D under the symmetries $S=C T_{x}, C T_{y}, O, C O^{\prime}$, $R_{x}$, and $R_{y}$.

\begin{tabular}{lcccccc}
\hline \hline$S$ & $C T_{x}$ & $C T_{y}$ & $O$ & $C O^{\prime}$ & $R_{x}$ & $R_{y}$ \\
\hline${ }^{S} A$ & $\mathrm{D}$ & $\mathrm{B}$ & $\mathrm{B}$ & $\mathrm{C}$ & $\mathrm{B}$ & $\mathrm{D}$ \\
${ }^{S} B$ & $\mathrm{C}$ & $\mathrm{A}$ & $\mathrm{C}$ & $\mathrm{B}$ & $\mathrm{A}$ & $\mathrm{C}$ \\
${ }^{S} C$ & $\mathrm{~B}$ & $\mathrm{D}$ & $\mathrm{D}$ & $\mathrm{A}$ & $\mathrm{D}$ & $\mathrm{B}$ \\
${ }^{S} D$ & $\mathrm{~A}$ & $\mathrm{C}$ & $\mathrm{A}$ & $\mathrm{D}$ & $\mathrm{C}$ & $\mathrm{A}$ \\
\hline \hline
\end{tabular}

TABLE IV. Transformation properties of the eight mixed phases A1, A2, B2, B3, C3, C4, D4, D1 under the symmetries $S=C T_{x}$, $C T_{y}, O, C O^{\prime}, R_{x}$, and $R_{y}$.

\begin{tabular}{lcccccc}
\hline \hline$S$ & $C T_{x}$ & $C T_{y}$ & $O$ & $C O^{\prime}$ & $R_{x}$ & $R_{y}$ \\
\hline${ }^{S} A 1$ & $\mathrm{D} 1$ & $\mathrm{~B} 3$ & $\mathrm{~B} 2$ & $\mathrm{C} 4$ & $\mathrm{~B} 3$ & $\mathrm{D} 1$ \\
${ }^{S} A 2$ & $\mathrm{D} 4$ & $\mathrm{~B} 2$ & $\mathrm{~B} 3$ & $\mathrm{C} 3$ & $\mathrm{~B} 2$ & $\mathrm{D} 4$ \\
${ }^{S} B 2$ & $\mathrm{C} 4$ & $\mathrm{~A} 2$ & $\mathrm{C} 3$ & $\mathrm{~B} 3$ & $\mathrm{~A} 2$ & $\mathrm{C} 4$ \\
${ }^{S}{ }_{B} 3$ & $\mathrm{C} 3$ & $\mathrm{~A} 1$ & $\mathrm{C} 4$ & $\mathrm{~B} 2$ & $\mathrm{~A} 1$ & $\mathrm{C} 3$ \\
${ }^{S} C 3$ & $\mathrm{~B} 3$ & $\mathrm{D} 1$ & $\mathrm{D} 4$ & $\mathrm{~A} 2$ & $\mathrm{D} 1$ & $\mathrm{~B} 3$ \\
${ }^{S} C 4$ & $\mathrm{~B} 2$ & $\mathrm{D} 4$ & $\mathrm{D} 1$ & $\mathrm{~A} 1$ & $\mathrm{D} 4$ & $\mathrm{~B} 2$ \\
${ }^{S} D 4$ & $\mathrm{~A} 2$ & $\mathrm{C} 4$ & $\mathrm{~A} 1$ & $\mathrm{D} 1$ & $\mathrm{C} 4$ & $\mathrm{~A} 2$ \\
${ }^{S}{ }^{S} 1$ & $\mathrm{~A} 1$ & $\mathrm{C} 3$ & $\mathrm{~A} 2$ & $\mathrm{D} 4$ & $\mathrm{C} 3$ & $\mathrm{~A} 1$ \\
\hline \hline
\end{tabular}


[1] J. C. Bednorz and K. A. Müller, Z. Phys. B: Condens. Matter 64, 189 (1986).

[2] D. S. Rokhsar and S. A. Kivelson, Phys. Rev. Lett. 61, 2376 (1988).

[3] P. W. Anderson, Science 235, 1196 (1987).

[4] S. Sachdev, Phys. Rev. B 40, 5204 (1989).

[5] L. S. Levitov, Phys. Rev. Lett. 64, 92 (1990).

[6] P. W. Leung, K. C. Chiu, and K. J. Runge, Phys. Rev. B 54, 12938 (1996).

[7] R. Moessner, S. L. Sondhi, and E. Fradkin, Phys. Rev. B 65, 024504 (2001).

[8] C. L. Henley, J. Phys.: Condens. Matter 16, S891 (2004).

[9] F. Alet, J. L. Jacobsen, G. Misguich, V. Pasquier, F. Mila, and M. Troyer, Phys. Rev. Lett. 94, 235702 (2005).

[10] F. Alet, Y. Ikhlef, J. L. Jacobsen, G. Misguich, and V. Pasquier, Phys. Rev. E 74, 041124 (2006).

[11] D. Charrier and F. Alet, Phys. Rev. B 82, 014429 (2010).

[12] J. Cano and P. Fendley, Phys. Rev. Lett. 105, 067205 (2010).

[13] A. F. Albuquerque and F. Alet, Phys. Rev. B 82, 180408(R) (2010).

[14] Y. Tang, A. W. Sandvik, and C. L. Henley, Phys. Rev. B 84, 174427 (2011).

[15] C. A. Lamas, A. Ralko, M. Oshikawa, D. Poilblanc, and P. Pujol, Phys. Rev. B 87, 104512 (2013).

[16] O. F. Syljuasen, Phys. Rev. B 71, 020401(R) (2005).

[17] O. F. Syljuasen, Phys. Rev. B 73, 245105 (2006).

[18] A. Ralko, D. Poilblanc, and R. Moessner, Phys. Rev. Lett. 100, 037201 (2008).

[19] D. Banerjee, M. Bögli, C. P. Hofmann, F.-J. Jiang, P. Widmer, and U.-J. Wiese, Phys. Rev. B 90, 245143 (2014).

[20] D. Horn, Phys. Lett. B 100, 149 (1981).

[21] P. Orland and D. Rohrlich, Nucl. Phys. B 338, 647 (1990).

[22] S. Chandrasekharan and U.-J. Wiese, Nucl. Phys. B 492, 455 (1997).
[23] G. 't Hooft, Nucl. Phys. B 153, 141 (1979).

[24] R. Moessner and K. S. Raman, Introduction to Frustrated Magnetism (Springer-Verlag, Berlin, Heidelberg, 2011), Chap. 17.

[25] Wei Zheng and Subir Sachdev, Phys. Rev. B 40, 2704(R) (1989).

[26] R. Moessner and S. L. Sondhi, Phys. Rev. Lett. 86, 1881 (2001).

[27] D. A. Ivanov, Phys. Rev. B 70, 094430 (2004).

[28] A. Ralko, M. Ferrero, F. Becca, D. Ivanov, and F. Mila, Phys. Rev. B 71, 224109 (2005).

[29] D. A. Huse, W. Krauth, R. Moessner, and S. L. Sondhi, Phys. Rev. Lett. 91, 167004 (2003).

[30] R. Moessner and S. L. Sondhi, Phys. Rev. B 68, 184512 (2003).

[31] M. Hermele, M. P. A. Fisher, and L. Balents, Phys. Rev. B 69, 064404 (2004).

[32] R. Moessner, S. L. Sondhi, and P. Chandra, Phys. Rev. Lett. 84, 4457 (2000).

[33] A. Ralko, M. Mambrini, and D. Poilblanc, Phys. Rev. B 80, 184427 (2009).

[34] G. Antonov, J. Chim. Phys. 5, 372 (1907).

[35] L. Balents, L. Bartosch, A. Burkov, S. Sachdev, and K. Sengupta, Phys. Rev. B 71, 144509 (2005).

[36] D. Poilblanc, F. Alet, F. Becca, A. Ralko, F. Trousselet, and F. Mila, Phys. Rev. B 74, 014437 (2006).

[37] A. Ralko, F. Mila, and D. Poilblanc, Phys. Rev. Lett. 99, 127202 (2007).

[38] S. Papanikolaou, E. Luijten, and E. Fradkin, Phys. Rev. B 76, 134514 (2007).

[39] H. Ribeiro, S. Bieri, and D. Ivanov, Phys. Rev. B 76, 172301 (2007).

[40] D. Poilblanc, Phys. Rev. Lett. 100, 157206 (2008).

[41] C. A. Lamas, A. Ralko, D. C. Cabra, D. Poilblanc, and P. Pujol, Phys. Rev. Lett. 109, 016403 (2012).

[42] T. M. Schlittler, T. Barthel, G. Misguich, J. Vidal, and R. Mosseri, Phys. Rev. Lett. 115, 217202 (2015).

[43] T. M. Schlittler, R. Mosseri, and T. Barthel, arXiv:1501.02242. 